\title{
Métodos globales de monitorización de la hemodinámica cerebral en el paciente neurocrítico: fundamentos, controversias y actualizaciones en las técnicas de oximetría yugular
}

\author{
M.A. Poca*; J. Sahuquillo*; R. Monforte** y A. Vilalta
}

Servicio de Neurocirugía*. Unidad de Cuidados Intensivos de Neurotraumatología**. Unidad de Investigación de Neurotraumatología. Hospital Universitario Vall d'Hebron. Universidad Autónoma de Barcelona.

Resumen

El papel relevante que la hipoxia tisular cerebral juega en la fisiopatología de los pacientes con un traumatismo craneoencefálico (TCE) justifica la necesidad de complementar la monitorización de estos pacientes con sistemas que aporten información sobre el flujo sanguíneo y el metabolismo cerebral. En la búsqueda de sistemas útiles en la cabecera del paciente, se han utilizado extrapolaciones del principio de Fick al encéfalo, utilizando métodos que estiman el flujo sanguíneo cerebral (FSC) a partir de la obtención de muestras de sangre del bulbo de la yugular. En los últimos años, las técnicas de oximetría yugular se han convertido en elementos de uso frecuente en las unidades que tratan pacientes con un TCE u otros pacientes neurocríticos, como los pacientes con una hemorragia subaracnoidea o con infartos masivos de la arteria cerebral media. El uso frecuente de estas técnicas en las últimas dos décadas, junto a la incorporación de otros sistemas de neuromonitorización, permiten en la actualidad matizar la información que estos métodos globales proporcionan y definir mejor tanto sus indicaciones como sus limitaciones. El objetivo de esta revisión es presentar los fundamentos y los conceptos básicos relacionados con la utilización clínica de las técnicas de oximetría yugular en el paciente neurocrítico. También presentamos y discutimos la evidencia más reciente que indica que determinadas variables, obtenidas de muestras de sangre del bulbo de la yugular, tales como las diferencias arterioyugulares de lactatos (AVDL) y el índice lactato-oxígeno (LOI), a pesar de su amplia utilización en la práctica clínica diaria, no ofrecen una información fiable sobre el metabolismo cerebral que permita la toma de decisiones terapéuticas.

PALABRAS CLAVE: Traumatismos craneoencefálicos. Flujo sanguíneo cerebral. Oximetría yugular. $\mathrm{AVDO}_{2}$. AVDL. LOI.

Recibido: 20-01-05. Aceptado: 09-02-05.
Global systems for monitoring cerebral hemodynamics in the neurocritical patient: basic concepts, controversies and recent advances in measuring jugular bulb oxygenation

Summary

Because of the importance of hypoxic/ischemic phenomena in head-injured patients, brain monitoring in these patients should be complemented by systems providing information on cerebral blood flow and metabolism. Indirect estimations of cerebral blood flow have been obtained from blood extracted from the jugular bulb, as a special bedside application of the Fick's principle to the brain. In the last few years, the use of jugular oximetry techniques has become routine in centers treating head-injured and other neurocritical patients such as those presenting subarachnoid hemorrhage or malignant middle cerebral artery infarction. The experience acquired in the use of these techniques, as well as the introduction of new neuromonitoring systems, have deepened our understanding of the information gained and have enabled more precise definition of

Abreviaturas. AVDL: Diferencias arterio-yugulares de lactato. $\mathrm{AVDO}_{2}$ : Diferencias arterio-yugulares de oxígeno. $\mathrm{CEO}_{2}$ : Coeficiente de extracción cerebral de oxígeno. $\mathrm{CO}_{2}$ : Dióxido de carbono. $\mathrm{CMRO}_{2}$ : Consumo metabólico cerebral de oxígeno. FiO Fracción inspirada de oxígeno. FSC: Flujo sanguíneo cerebral. Hb: Hemoglobina. HSA: Hemorragia subaracnoidea. LCR: Líquido cefalorraquideo. LOI: Índice lactato-oxígeno. $\mathrm{NO}_{2}$ : Óxido nitroso. $\mathrm{O}_{2}$ : Oxígeno. PaCO${ }_{2}$ : Presión arterial de dióxido de carbono. $\mathrm{PaO}_{2}$ : Presión arterial de oxígeno. PAM: Presión arterial media. PIC: Presión intracraneal. PPC: Presión de perfusión cerebral. $\mathrm{PtiO}_{2}$ : Presión tisular de oxígeno. $\mathrm{SjO}_{2}$ : Saturación de la oxihemoglobina en el bulbo de la yugular. SPECT: Tomografía computarizada por emisión de positrones simple. TC: Tomografia computarizada. TCDB: Traumatic Coma Data Bank. TCE: Traumatismo craneoencefálico. TCEG: Traumatismo craneoencefálico grave. UCI: Unidad de cuidados intensivos. 
their indications and limitations. This review describes the basic concepts underlying the use of jugular oximetry techniques in the neurocritical patient. We also explain the reasons why several variables derived from jugular blood such as arterio-jugular differences of lactate (AVDL) or the lactate-oxygen index (LOI) do not provide accurate information on brain metabolism.

KEY WORDS: Head injury. Cerebral blood flow. Jugular oximetry. $\mathrm{AVDO}_{2}$. AVDL. LOI.

"It seemed theoretically correct that if one could study the blood directly before it reached the brain, and then could study it directly as it came from the brain, without admixture with venous blood from other parts of the body, something might be learned of what takes place within the brain"

Myerson et al., 1927

En 1945, Kety y Smith fueron los primeros en aplicar el principio de Fick al encéfalo y cuantificaron el flujo sanguineo cerebral a partir de las diferencias arterioyugulares de oxígeno. Las técnicas de oximetría yugular que se exponen en este capitulo se fundamentan en este principio.

\section{Introducción}

La mortalidad de los pacientes que han presentado un traumatismo craneoencefálico (TCE) grave ha disminuido de forma importante en los últimos años, sin que este descenso se haya acompañado de un aumento significativo del número de pacientes que quedan en estado vegetativo o gravemente incapacitados ${ }^{2,6,37,39,79}$. Este hecho se debe fundamentalmente al mejor conocimiento de la fisiopatología de las lesiones neurotraumáticas y a la aplicación de protocolos de neuromonitorización y tratamiento más específicos y sistematizados ${ }^{6,39,82,119,122,127,139}$. Las guías de práctica clínica de la Brain Trauma Foundation indican que la presión intracraneal (PIC) constituye el único parámetro encefálico que debe monitorizarse de forma obligada en los pacientes con un TCE grave y que presentan una TC cerebral patológica ${ }^{139}$. Sin embargo, en el momento actual existe cava vez más evidencia de la necesidad de complementar la neuromonitorización de estos pacientes con la medición de otras variables que aporten información adicional sobre diversos aspectos del flujo sanguíneo y/o del metabolismo cerebral $1^{7,8,143}$.

A pesar de que en el momento actual el flujo sanguíneo y el metabolismo cerebral pueden cuantificarse por sistemas altamente sofisticados (PET, Xenon-TC, RM...), en la práctica clínica los pacientes que han presentado un TCE grave requieren la aplicación de sistemas que puedan utili- zarse en la cabecera del enfermo y que ofrezcan, a ser posible, una información continua sobre estos parámetros. En las últimas décadas hemos estimado el valor del flujo sanguíneo cerebral (FSC) y ciertos aspectos del metabolismo del encéfalo, a partir de los valores de oxihemoglobina y de las concentraciones de lactato obtenidos en el bulbo de la vena yugular interna, como aplicaciones especiales del principio de Fick al encéfalo ${ }^{18,21,46,111}$. En el momento actual estas técnicas se han convertido en instrumentos de uso cotidiano en la neuromonitorización de los pacientes con un TCE o en otros tipos de pacientes neurocríticos ${ }^{52,62}$.

Sin embargo, después de una amplia utilización clínica de las técnicas de oximetría yugular y de la aplicación simultánea de nuevos sistemas de monitorización cerebral, hemos aprendido a matizar la información que nos ofrecen y a establecer de manera más adecuada sus indicaciones y limitaciones. Del mismo modo, también hemos objetivado que algunas de las variables que obteníamos a partir de muestras de sangre yugular, como las AVDL o el LOI, no nos ofrecen una información fiable sobre los procesos metabólicos que tienen lugar en el encéfalo, por lo que no deberían ser consideradas en la toma de decisiones terapéuticas en los pacientes neurocríticos ${ }^{102}$.

En esta revisión pretendemos exponer los fundamentos, la situación actual y las indicaciones y limitaciones de las técnicas de oximetría yugular en la monitorización encefálica de los pacientes neurocríticos. Se expondrán los fundamentos que confirman que las variables calculadas a partir de la medición de lactato en el bulbo de la vena yugular (AVDL y LOI) no ofrecen una información válida sobre el metabolismo cerebral. También se revisarán diversos aspectos prácticos relacionados con el uso clínico de las técnicas de oximetría yugular. Finalmente, se expondrá la utilidad de estas técnicas para determinar la preservación o alteración de los mecanismos de control del FSC.

\section{LAS TÉCNICAS DE OXIMETRÍA YUGULAR COMO MÉTO- DOS DE ESTUDIO DEL FSC}

\section{Fundamentos de las técnicas de oximetría yugular}

La utilización de las técnicas de oximetría yugular como métodos indirectos de estudio del FSC se fundamenta en los trabajos de Adolf Fick ${ }^{40}$. En 1870, este autor calculó el gasto cardíaco a partir de la relación entre el volumen de oxígeno absorbido cada minuto por los pulmones y las diferencias arteriovenosas de oxígeno ${ }^{40}$. Posteriormente, y como aplicaciones especiales del principio de Fick, se estimó el flujo sanguíneo de determinados órganos a partir de métodos de dilución de un "indicador". Krogh y Lindhard en $1912^{68}$ fueron los primeros en utilizar el óxido nitroso para medir el flujo hemático a través de los pulmones y, de aquí, el gasto cardíaco. Kety y Schmidt ${ }^{63}$ aplicaron 
el principio de Fick para estimar cuantitativamente el FSC en humanos. Estos autores determinaron el FSC y el consumo de oxígeno cerebral $\left(\mathrm{CMRO}_{2}\right)$ en 14 varones jóvenes y sanos, obteniendo un valor medio de $54 \pm 12 \mathrm{~mL} / 100$ $\mathrm{g} / \mathrm{min}$ y $1.5 \mu \mathrm{moL} / \mathrm{g} / \mathrm{min}$ respectivamente ${ }^{63}$.

En el estudio de la hemodinámica y del metabolismo cerebral a partir de técnicas de oximetría yugular destacan, también, los estudios clásicos de Myerson ${ }^{92}$ y Gibbs $^{46}$. Myerson afirmó que si se estudiaba el contenido hemático directamente en el punto de entrada de la sangre al encéfalo (punción directa en la arteria carótida interna) y en el punto de salida, sin que se mezclara sangre procedente de otras partes del organismo (punción en la vena yugular interna ipsilateral antes de recibir ramas afluentes de la cara y cuello), podían conocerse los acontecimientos metabólicos que tenían lugar en el cerebro ${ }^{92}$. Myerson sugería, además, que si simultáneamente se extraía una muestra de líquido cefalorraquídeo (LCR) espinal, podía obtenerse una información completa sobre el metabolismo cerebral ${ }^{92}$.

Gibbs y col. en $1942^{46}$, basándose en los postulados de Myerson, determinaron los valores normales de algunos elementos de la sangre arterial y de la sangre de la vena yugular interna, obtenida en ambos casos por punción directa (oxígeno, dióxido de carbono, glucosa, ácido láctico, potasio inorgánico, PH y saturación de oxígeno). Para tal efecto, examinaron a 50 estudiantes de medicina voluntarios, sanos, varones y con una edad comprendida entre los 18 y 29 años. Antes de obtener las muestras hemáticas, comprobaron que no presentaban signos ni síntomas de patología neurológica y que el estudio electroencefalográfico era normal ${ }^{46}$. En el momento actual, los valores obtenidos por estos autores continúan utilizándose como valores de referencia.

\section{Aplicación del principio de Fick al encéfalo}

Kety y Schmidt ${ }^{64}$ fueron los primeros en estimar cuantitativamente el FSC en humanos, utilizando el óxido nitroso $\left(\mathrm{N}_{2} \mathrm{O}\right)$ como indicador y calculando el valor de las diferencias arterio-yugulares de este gas. $\mathrm{El}_{2} \mathrm{O}$ es un gas metabólicamente inerte que difunde de forma rápida al encéfalo. Estos autores administraban $\mathrm{N}_{2} \mathrm{O}$ de forma inhalada junto al aire inspirado y determinaban su concentración en muestras hemáticas arteriales y yugulares obtenidas de forma secuencial ${ }^{64}$. En el momento actual, y con los mismos fundamentos, se utilizan Xenón, Kriptón u otros indicadores para cuantificar el valor del FSC. Sin embargo, las determinaciones del FSC a través de estas técnicas o de las que combinan el uso de trazadores con técnicas tomográficas (PET, SPECT) son en general complejas y no pueden realizarse de forma continua. En la práctica clínica, el diagnóstico de las lesiones isquémicas requiere un método relativamente simple y que permita estudios repetitivos, e incluso continuos, de la hemodinámica cerebral.
Simplificando el método de Kety y Schmidt, estudios experimentales y clínicos practicados por autores clásicos y validados por estudios más recientes indicaron que el FSC también podía estimarse, en determinadas condiciones, a partir de la medición de las diferencias arterio-yugulares de oxígeno $\left(\mathrm{AVDO}_{2}\right)$ o de otras variables hemometabólicas derivadas del valor de la oxihemoglobina obtenida a nivel del bulbo de la vena yugular interna (extracción cerebral de $\mathrm{O}_{2}$ o saturación de la oxihemo-globina yugular) $18,25,30,31,45,46,109,111$. Las primeras determinaciones de la saturación yugular de oxígeno $\left(\mathrm{SjO}_{2}\right)$ se obtuvieron durante los años 1930 y 1940, a partir de punciones directas de la porción superior de la vena yugular interna $(1 \mathrm{~cm}$ por delante y por debajo de la apófisis mastoides). Posteriormente, las punciones directas se sustituyeron por la colocación de un catéter retrógrado, que permitía realizar determinaciones seriadas sin practicar nuevas punciones yugulares ${ }^{47}$. En las últimas décadas, la incorporación a esta técnica de sistemas de fibra óptica nos ha permitido obtener información continua sobre la hemodinámica cerebral ${ }^{31}$.

El principio básico de la medición de la $\mathrm{SjO}_{2}$ a través de catéteres de fibra óptica radica en que la oxihemoglobina absorbe la luz de forma diferente a la desoxihemoglobina ${ }^{30}$. El catéter incorpora dos fibras ópticas que emiten luz en dos o tres longitudes de onda dependiendo del sistema: Oximetrix 3 (suministrado por Abbot Laboratories, S.A., Madrid) utiliza tres longitudes de onda y Baxter (suministrado por Baxter S.A., Valencia) dos. La señal luminosa accede al torrente sanguíneo a través de la fibra óptica incidente, se refleja y retorna al monitor a través de la segunda fibra óptica del catéter. En el monitor, un detector fotoeléctrico cuantifica la cantidad de luz absorbida y determina el porcentaje de oxihemoglobina en relación con la hemoglobina total. Cuando el catéter sólo utiliza dos longitudes de onda el valor de la hemoglobina debe introducirse de forma manual. En cambio, en los catéteres con tres longitudes de onda este paso no es necesario, ya que el monitor es capaz de estimar la concentración de hemoglobina a partir de las diferentes absorciones de luz. El inconveniente fundamental de estos sistemas es que, dado que inicialmente no fueron diseñados para su uso a nivel yugular, presentan más artefactos que cuando se utilizan para su uso habitual (arteria pulmonar o arteria umbilical), lo que obliga a realizar calibraciones periódicas del sistema ${ }^{31,41,93,108,133}$. No obstante, los nuevos catéteres diseñados de forma específica para la monitorización del bulbo de la yugular requieren menos calibraciones y son más fiables, tanto en la monitorización de pacientes ingresados en la UCI como en quirófano.

\section{Monitorización de la hemodinámica cerebral a partir de las $\mathrm{AVDO}_{2}$}

El principio de Fick permite el cálculo del FSC a 
partir de la relación entre el $\mathrm{CMRO}_{2}$ y las $\mathrm{AVDO}_{2}$ : (FSC $\left.=\mathrm{CMRO}_{2} / \mathbf{A V D O}_{2}\right)$. En condiciones normales el flujo sanguíneo aumenta o disminuye en función de los requerimientos metabólicos tisulares. En esta situación existe un perfecto acoplamiento entre el FSC y el $\mathrm{CMRO}_{2}$, que hace que las $\mathrm{AVDO}_{2}$ permanezcan constantes ${ }^{111}$. Sin embargo, un gran número de pacientes con un TCE presentan una alteración de los mecanismos reguladores del $\mathrm{FSC}^{10,36,50,94 \text {, }}$ 117,121,123, de modo que pueden presentar un FSC disminuido, normal o elevado, independientemente de la reducción del $\mathrm{CMRO}_{2}{ }^{69,97}$. En este contexto la relación entre $\mathrm{CMRO}_{2} \mathrm{y}$ FSC puede variar y los valores de las $\mathrm{AVDO}_{2}$ constituyen una estimación indirecta del FSC. En general se acepta que: a) unas $\mathrm{AVDO}_{2}$ normales sugieren un correcto acoplamiento entre FSC y $\mathrm{CMRO}_{2}$ (normoperfusión), b) unas $A V D O$, bajas indican que el FSC es excesivo respecto a los requerimientos metabólicos cerebrales (hiperemia) y c) unas AVDO2 elevadas nos informan sobre una disminución del FSC (hipoperfusión), ya que el cerebro compensa el descenso de flujo sanguíneo a través de una mayor extracción de $\mathrm{O}_{2}^{23,24,110,111}$.

El cálculo de las $\mathrm{AVDO}_{2}$ requiere determinaciones de la concentración de hemoglobina $(\mathrm{Hb})$, de la saturación arterial de $\mathrm{O}_{2}\left(\mathrm{SaO}_{2}\right)$, de la saturación de $\mathrm{O}_{2}$ en el bulbo de la yugular $\left(\mathrm{SjO}_{2}\right)$ y el valor de las presiones parciales de $\mathrm{O}_{2}$ a nivel arterial $\left(\mathrm{PaO}_{2}\right)$ y yugular $\left(\mathrm{PjO}_{2}\right)$, de acuerdo con la siguiente ecuación ${ }^{30}$ :

$\mathbf{A V D O}_{2}=\mathbf{1 . 3 4} * \mathbf{H b}\left[\left(\mathbf{S a O}_{2}-\mathbf{S j O}_{2}\right) / \mathbf{1 0 0}\right]+\left(\mathrm{PaO}_{2}-\mathrm{PjO}_{2}\right) * 0,0031$

En esta ecuación pueden diferenciarse dos componentes: el primero, que incluye la $\mathrm{Hb}$ y las saturaciones de la oxihemoglobina arterial y yugular, y el segundo en el que se contempla el valor del oxígeno disuelto, no asociado a la $\mathrm{Hb}$, que contiene la sangre arterial y la sangre yugular. Sin embargo, a efectos prácticos se prescinde del segundo componente de la fórmula, ya que la aportación de $\mathrm{O}_{2}$ a nivel celular que procede del $\mathrm{O}_{2}$ plasmático no asociado a la $\mathrm{Hb}$ es muy reducida y se considera prácticamente despreciable.

El resultado de esta ecuación viene dado en volumen por 100. Es decir, $\mathrm{mL}$ de $\mathrm{O}_{2}$ por $100 \mathrm{~g}$ de tejido y por minuto. Sin embargo, si aplicamos un factor de corrección $(0,446)$ el resultado final se expresa en $\mu \mathrm{moL} / \mathrm{mL}$, que son las unidades que utilizan diversos autores en la literatura $^{109,111}$. Los valores que hoy consideramos como normales de las $\mathrm{AVDO}_{2}$ proceden fundamentalmente de los trabajos de Gibbs y col. de los años cuarenta, en los que, como hemos comentado, realizaron punciones directas a nivel arterial y en la porción más craneal de la vena yugular interna en un grupo de 50 voluntarios sanos ${ }^{46}$. A partir de estos estudios se establecieron como normales valores de las $\mathrm{AVDO}_{2}$ situados entre 4 - $8 \mathrm{Vol} \%(1.3-3 \mu \mathrm{moL} / \mathrm{mL})$; valores de las $\mathrm{AVDO}_{2}$ por debajo de $4 \mathrm{Vol} \%(<1.3 \mu \mathrm{moL} /$ $\mathrm{mL}$ ) indicarían que el FSC está aumentado (hiperemia) y cuando los valores de las $\mathrm{AVDO}_{2}$ fueran superiores a $8 \mathrm{Vol}$ $\%$ ( $>3 \mu \mathrm{moL} / \mathrm{mL})$ indicarían que existe una reducción del FSC (hipoperfusión) ${ }^{46}$.

El coeficiente de extracción de $\mathrm{O}_{2}\left(\mathrm{CEO}_{2}\right)$ y la saturación de la oxihemoglobina yugular $\left(\mathrm{SjO}_{2}\right)$ como parámetros alternativos en la monitorización de la hemodinámica cerebral

Dado que la capacidad de transporte de oxígeno de la hemoglobina es la misma cuando se encuentra en sangre arterial o en sangre venosa, Cruz y col. ${ }^{17,20,23,24}$ simplificaron el método alegando que los cambios en la extracción de oxígeno por parte del cerebro vienen igualmente representados simplemente por las modificaciones en las diferencias de saturación de la oxihemoglobina arterio-yugular. Este autor denominó a la nueva variable "Coeficiente de Extracción de Oxígeno" $\left(\mathrm{CEO}_{2}=\mathrm{SaO}_{2}-\mathrm{SjO}_{2}\right)$ y sustituyó las $\mathrm{AVDO}_{2}$ por el $\mathrm{CEO}_{2}$ en la fórmula general de la dinámica hemometabólica cerebral ${ }^{25}$ :

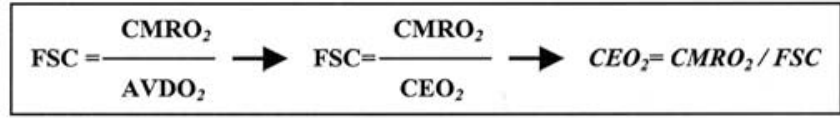

El segundo argumento de Cruz para la introducción de esta nueva variable fue que, en casos de anemia, las $\mathrm{AVDO}_{2}$ pueden enmascarar situaciones isquémicas ${ }^{22}$. En la anemia aguda, la disminución de la hemoglobina puede condicionar descensos "artificiales" de las $\mathrm{AVDO}_{2}$. Estos descensos simularían situaciones de normoperfusión o estados hiperémicos, cuando en realidad podría existir un compromiso en la oxigenación cerebral global. Julio Cruz sugería que en casos de anemia importante los acontecimientos metabólicos cerebrales quedarían mejor reflejados a partir del cálculo del $\mathrm{CEO}_{2}^{22}$.

Cuando existe un consumo metabólico cerebral de oxígeno constante, el $\mathrm{CEO}_{2}$ estima de manera indirecta el valor del $\mathrm{FSC}$. Al igual que ocurría con las $\mathrm{AVDO}_{2}$, la relación entre el FSC y el $\mathrm{CEO}_{2}$ se establece de forma inversa: cuando el FSC aumenta, el $\mathrm{CEO}_{2}$ se reduce y cuando el FSC disminuye, el $\mathrm{CEO}_{2}$ aumenta. El rango de normalidad para este parámetro se ha establecido también a partir de los estudios de Gibbs y col. ${ }^{46}$, situándose entre un $24 \%$ y un $40 \%$. Valores comprendidos dentro de este rango indican un correcto acoplamiento entre metabolismo y FSC. Valores inferiores al 24\% sugieren una hiperperfusión cerebral global con respecto al consumo metabólico de oxígeno (hiperemia o perfusión de lujo). Por último, valores superiores al 40\% reflejan una situación de hipoperfusión cerebral global relativa al $\mathrm{CMRO}_{2}$ (hipoxia cerebral oliguémica $)^{46}$. 


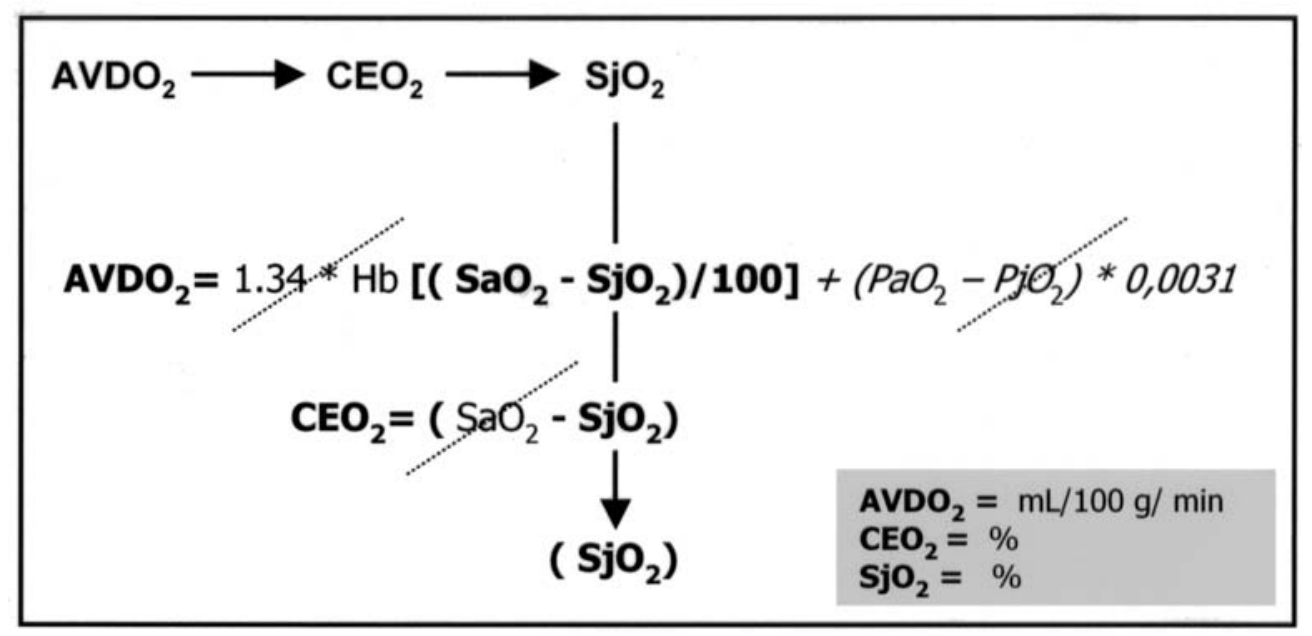

Figura 1. Fórmulas para el cálculo de los diversos parámetros utilizados en las técnicas de oximetría yugular. La figura muestra cómo a partir de las $A V D O_{2}$, y prescindiendo de la hemoglobina y del contenido hemático de $\mathrm{O}_{2}$ disuelto, se obtiene el $\mathrm{CEO}_{2}$. Prescindiendo, además, del valor de la saturación arterial de $\mathrm{O}_{2}$, podemos utilizar la $\mathrm{SjO}_{2}$ como método de estimación del FSC. Las $\mathrm{AVDO}_{2}$ y el $\mathrm{CEO}_{2}$ presentan una relación inversa con los valores del FSC, mientras que la $\mathrm{SjO}_{2}$ se relaciona de forma directa con el FSC.

A pesar de que el $\mathrm{CEO}_{2}$ facilita los cálculos, el parámetro más utilizado en la clínica para estimar el FSC es la determinación simple del valor de la saturación de la oxihemoglobina yugular $\left(\mathrm{SjO}_{2}\right)^{30,56,108}$. $\mathrm{La} \mathrm{SjO}_{2}$ muestra el porcentaje de hemoglobina oxigenada respecto a la hemoglobina total, mostrando el oxígeno que capta el encéfalo de la sangre arterial e indicándonos de modo indirecto el valor del FSC. $\mathrm{La} \mathrm{SjO}_{2}$ refleja el balance entre el aporte y el consumo cerebral de $\mathrm{O}_{2}^{30,54}$. En cambios paralelos entre aporte y consumo la $\mathrm{SjO}_{2}$ se mantiene constante. Cuando el aporte es insuficiente, o las necesidades son excesivas, la $\mathrm{SjO}_{2}$ desciende. Cuando el aporte supera el consumo, o cuando el consumo está muy reducido, $\mathrm{la} \mathrm{SjO}_{2}$ aumenta. Por tanto, asumiendo un contenido arterial de oxígeno y un $\mathrm{CMRO}_{2}$ constantes, los cambios en la $\mathrm{SjO}_{2}$ reflejarán cambios directamente proporcionales en el FSC. La figura 1 resume el origen de las diversas variables que pueden utilizarse para estimar el valor del FSC y que se calculan a partir de muestras hemáticas obtenidas en el bulbo de la vena yugular interna.

\section{Valores normales de $\mathrm{la}_{\mathrm{SjO}_{2}}$. Umbrales de isquemia e hiperemia}

Los valores normales de la $\mathrm{SjO}_{2}$ que hemos utilizado durante años proceden también de los estudios de Gibbs y $\mathrm{col}^{46}$. En el caso de la $\mathrm{SjO}_{2}$, el rango de normalidad quedó establecido entre un $55 \%$ y un $71 \%$, con un valor medio del $61.8 \%{ }^{46}$. Estos valores son superiores a la saturación venosa mixta, lo que indica que en condiciones normales el cerebro extrae más oxígeno que otros órganos. En
1963, Datsur y col. ${ }^{27}$ estudiaron la influencia de la edad en la circulación y metabolismo cerebrales en el hombre, obteniendo valores de la $\mathrm{SjO}_{2}$ muy similares a los de Gibbs y col. En este segundo estudio, practicado en 26 ancianos voluntarios sin patología neurológica a los que se implantó un catéter yugular retrógrado, los límites inferior, superior y el valor medio de la $\mathrm{SjO}_{2}$ fueron $55 \%, 74 \%$ y $64 \%$ respectivamente ${ }^{27}$.

Recientemente, Chieregato y col. ${ }^{9}$ han publicado un nuevo estudio, practicado en 12 pacientes afectos de una enfermedad de Cushing de inicio reciente y sin hipertensión arterial establecida, que objetiva que los valores normales de la $\mathrm{SjO}_{2}$ pueden ser inferiores a los que indicaban los estudios previos. A diferencia de los estudios iniciales, en los que las muestras de sangre yugular se obtuvieron por punción directa ${ }^{46} \mathrm{o}$ a través de un catéter sin control radiológico ${ }^{27}$, Chieregato y col. obtuvieron las muestras yugulares bajo visión directa de la ubicación de la punta del catéter (angiografía digital), a una velocidad de extracción muy lenta $(1 \mathrm{~mL} / \mathrm{min})$ y midieron la saturación de oxígeno de la hemoglobina con un co-oxímetro, obteniendo un valor de $\mathrm{SjO}_{2}$ medio de 57,1\% (intervalo de confianza [IC] del 95\%: 52,3 - 61,6), con un límite inferior del 44,7\% (IC del 95\%: 36,5 - 53,0) y un límite superior

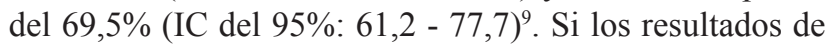
estos autores se confirman en una serie de pacientes más amplia, el umbral de isquemia de la $\mathrm{SjO}_{2}$ debería descender hasta al menos el 46\%, con las implicaciones diagnósticas, pronósticas y terapéuticas que esta redefinición de "normalidad" implica.

Los umbrales de isquemia para este parámetro se obtu- 


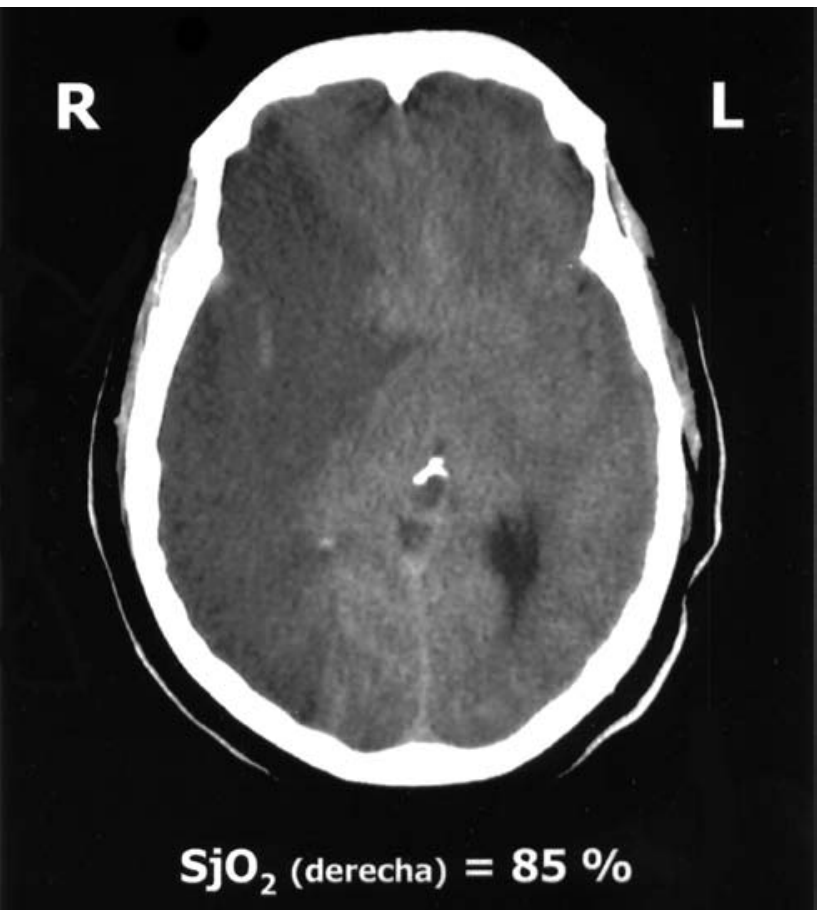

Figura 2. Paciente con un infarto del territorio de la arteria cerebral media derecha. La imagen muestra cómo, a pesar del extenso territorio necrótico, el valor de la $\mathrm{SjO}_{2}$ se encuentra dentro del rango de la "hiperemia" (85\%). Esta discrepancia puede explicarse porque el paciente presenta una reducción del FSC en el área de infarto, al mismo tiempo que en este hemisferio existe una importante reducción del consumo cerebral de $\mathrm{O}_{2}$. vieron a partir de estudios experimentales, que establecieron que por debajo de una $\mathrm{SjO}_{2}$ del $45 \%$ aparece confusión y por debajo del $24 \%$ se produce una pérdida de conciencia. En los TCE el valor medio de la $\mathrm{SjO}_{2}$ es superior al normal y el intervalo de distribución es más amplio que en los individuos normales. Sin embargo, diversos estudios clínicos y experimentales han constatado que en los TCE caídas de la $\mathrm{SjO}_{2}$ por debajo del $50 \%$ se correlacionan con un peor pronóstico ${ }^{30,51,106,109,133,146}$. Estos resultados, junto con la conocida susceptibilidad del encéfalo traumatizado a los insultos isquémicos ${ }^{34}$, sugieren que en los TCE los valores de la $\mathrm{SjO}_{2}$ deberían mantenerse siempre por encima del $50 \% \mathrm{y}$, de forma óptima, por encima del $55 \%$ o incluso del $60 \%{ }^{119}$. No obstante, el umbral de isquemia que utilizamos en los TCE no es válido en los pacientes con un infarto cerebral extenso. En los pacientes con un infarto extenso del territorio de la arteria cerebral media pueden coexistir valores normales o elevados de la $\mathrm{SjO}_{2}$ en el contexto de un área cerebral necrótica muy extensa ${ }^{61}$. La figura 2 muestra las imágenes radiológicas y los valores de la $\mathrm{SjO}_{2}$ de un paciente con un infarto maligno de la arteria cerebral media.

De acuerdo con el estudio clásico de Gibbs y col. ${ }^{46}$, el umbral de hiperemia para la $\mathrm{SjO}_{2}$ se sitúa por encima del $71 \%$. Aplicado al contexto de los pacientes con un TCE, este valor asciende al 75\%. Valores de la $\mathrm{SjO}_{2}>75 \%$ indicarían que el aporte de oxígeno al encéfalo supera el consumo tisular de este substrato metabólico. Sin embargo, esta situación aparentemente ideal se encuentra de forma más frecuente en los pacientes que presentan un mal resul-

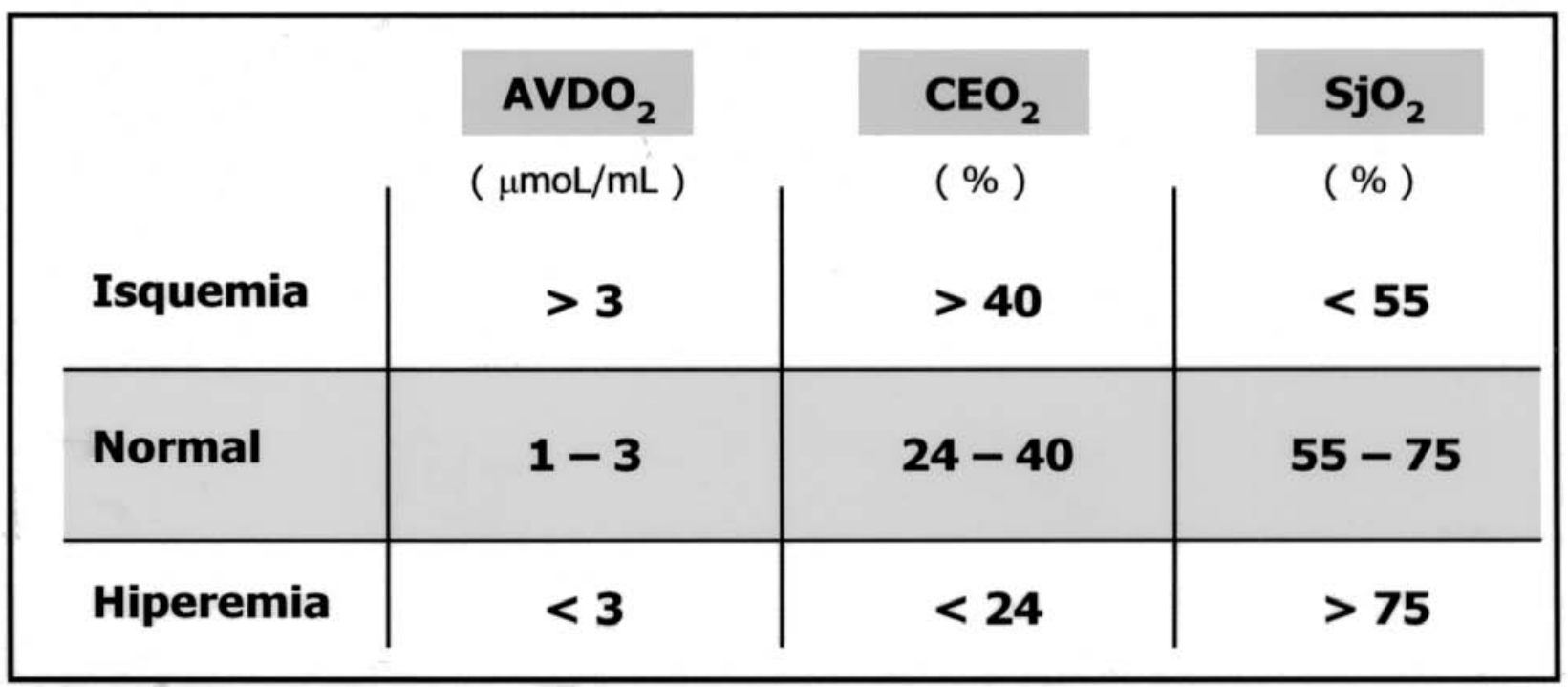

Figura 3. Valores aceptados como normales y como umbrales de isquemia e hiperemia para las $\mathrm{AVDO}_{2}, \mathrm{CEO}_{2}$ y la $\mathrm{SjO}_{2}$ en pacientes con un TCE. 
tado final ${ }^{14,75}$. Este hecho sugiere que en los pacientes con un TCE también debería investigarse el origen de los valores de $\mathrm{SjO}_{2}$ superiores al $75 \%$. La figura 3 resume los valores de normalidad y los umbrales de isquemia e hiperemia definidos para las $\mathrm{AVDO}_{2}$, el $\mathrm{CEO}_{2}$ y la $\mathrm{SjO}_{2}$ en individuos normales y en pacientes con un TCE.

\section{Significado de los valores de $\mathrm{SjO}_{2}$ superiores al $75 \%$}

De acuerdo con los parámetros aceptados en la literatura, valores de la $\mathrm{SjO}_{2}>75 \%$ se atribuyen a priori a una situación de hiperemia cerebral, en la que el aporte de $\mathrm{O}_{2}$ supera las demandas metabólicas del encéfalo. Sin embargo, la $\mathrm{SjO}_{2}$ es directamente proporcional al valor del FSC pero inversamente proporcional al consumo metabólico cerebral de $\mathrm{O}_{2}$, por lo que un valor elevado de este parámetro puede indicarnos que el paciente presenta un aumento real del FSC (hiperemia) o un descenso del consumo metabólico de $\mathrm{O}_{2}$, no acoplado a una reducción del FSC, como puede ocurrir en algunos pacientes con un TCE o en situaciones de hipotermia o coma barbitúrico. También podemos encontrar valores elevados de la $\mathrm{SjO}_{2}$ en pacientes que presentan grandes lesiones isquémicas cerebrales o que se encuentran en una situación de isquemia global con necrosis tisular importante. En estos casos la reducción del FSC se acompaña de una reducción del consumo metabólico de $\mathrm{O}_{2}$, por lo que la $\mathrm{SjO}_{2}$ puede ser normal o incluso elevada, sin que indique la presencia de la isquemia cerebral.

En determinadas situaciones puede existir una mayor afinidad entre la hemoglobina y el $\mathrm{O}_{2}$, de lo que resulta una mayor dificultad en la cesión de $\mathrm{O}_{2}$ a los tejidos y valores de la $\mathrm{SjO}_{2}$ elevados pueden sugerir un estado de hiperemia, cuando en realidad existe un compromiso en la liberación de $\mathrm{O}_{2}$. Por otra parte, los pacientes en situación de muerte cerebral suelen presentar valores de la $\mathrm{SjO}_{2}$ muy elevados $\mathrm{y}$, en algunos casos, cercanos al $100 \%{ }^{35,146}$. En estos pacientes, además de la falta de FSC, los valores altos de la $\mathrm{SjO}_{2}$ se deben a la contaminación extracerebral de las muestras yugulares $^{146}$. De este modo, los valores de $\mathrm{SjO}_{2}>75 \%$ pueden corresponder situaciones fisiopatológicas diversas y pueden llegar a requerir maniobras terapéuticas muy distintas.

En un estudio en el que se cuantificaron de manera simultánea el valor de la $\mathrm{SjO}_{2}$, el volumen del FSC (calculado a partir del método de Kety-Schmidt utilizando $\mathrm{N}_{2} \mathrm{O}$ como trazador) y la PPC se objetivó que no existía correlación entre estas variables ${ }^{14}$. Los pacientes con valores de $\mathrm{SjO}_{2}>75 \%$ presentaron valores de FSC y PPC muy variables $(6,7-195 \mathrm{~mL} / 100 \mathrm{~g} / \mathrm{min}$ y $20-119 \mathrm{mmHg}$, respectivamente $)^{14}$. Este mismo estudio objetivó que algunos de estos pacientes presentaban un consumo metabólico de $\mathrm{O}_{2}$ muy reducido $^{14}$. Esta heterogeneidad fisiopatológica asociada a valores de $\mathrm{SjO}_{2}>75 \%$ también ha sido demostrada por otros autores ${ }^{29,73}$ y orienta a la necesidad de complementar las técnicas de oximetría yugular con la información que ofrecen otros métodos diagnósticos.

\section{Otros parámetros derivados de determinaciones arte- rio-yugulares: las diferencias arterio-yugulares de lactatos (AVDL) y el índice lactato oxígeno (LOI).}

En 1987, Robertson y col. ${ }^{110}$ introdujeron nuevos conceptos que modificaron la interpretación clásica de las $\mathrm{AVDO}_{2}$. Estos autores encontraron que en situación de bajo FSC inicialmente el $\mathrm{CMRO}_{2}$ permanecía constante, gracias a un incremento en el índice de extracción de oxígeno. Si el FSC seguía disminuyendo, el efecto compensador era insuficiente y aparecía isquemia: el $\mathrm{CMRO}_{2}$ caía y se incrementaba la producción cerebral de lactatos. Según sus resultados, cuando el $\mathrm{CMRO}_{2}$ era inferior a $0,6 \mu \mathrm{moL} / \mathrm{g} /$ min y simultáneamente la producción cerebral de lactatos era superior a $0,06 \mu \mathrm{moL} / \mathrm{g} / \mathrm{min}$ aparecían lesiones cerebrales isquémicas de carácter irreversible ${ }^{110}$. Desde un punto de vista teórico, la determinación de muestras simultáneas a nivel arterial y en el bulbo de la yugular también podían utilizarse para calcular una nueva variable: las diferencias arterio-yugulares de lactatos $(\boldsymbol{A} \boldsymbol{V D} \boldsymbol{L})^{110}$. De acuerdo con Robertson y col. las AVDL permitían analizar la situación del metabolismo anaerobio, ya que podían detectar aumentos en la producción cerebral de lactatos ${ }^{110}$.

El mismo grupo de investigadores, en un trabajo posterior, calculó la cantidad de glucosa que se metabolizaba por la vía anaerobia y por la vía aerobia, estableciendo el índice lactato-oxígeno (LOI) a través de la relación negativa entre las AVDL y las $\mathrm{AVDO}_{2}\left(\boldsymbol{L O I}=-\boldsymbol{A V} \boldsymbol{D} \boldsymbol{L} / \boldsymbol{A V D O} \boldsymbol{O}_{2}\right)^{111}$. El valor normal del LOI quedó establecido por debajo de 0,03 . Un LOI $\geq 0,08$ era indicativo de un incremento en la producción cerebral de lactatos $\mathrm{y}$, por tanto, de una situación de isquemia-infarto o de una hipoperfusión lo suficientemente importante como para alterar el metabolismo cerebral ${ }^{111}$. A partir de mediciones paralelas del FSC a través de la técnica del $\mathrm{N}_{2} \mathrm{O}$ de Kety-Schmidt y de las $\mathrm{AVDO}_{2}$-AVDL, el grupo de Robertson encontró que en situación de isquemiainfarto cerebral $(\mathrm{LOI} \geq 0,08)$, las $\mathrm{AVDO}_{2}$ eran variables y podían no estimar los verdaderos valores del FSC ${ }^{111}$.

De los estudios de estos investigadores surgió una clasificación del FSC, basada en las estimaciones de las $\mathrm{AVDO}_{2}$, de las AVDL y del LOI, en la que se distinguían cuatro patrones: 1) Isquemia-infarto: $\mathrm{LOI} \geq 0,08$ y $\mathrm{AVDO}_{2}$ variables; 2) Hipoperfusión compensada: $\mathrm{AVDO}_{2}$ elevadas $(>3,0 \mu \mathrm{moL} / \mathrm{mL})$ y LOI normal $(<0,03) ; 3) \boldsymbol{F S C}$ normal: LOI normal y $\mathrm{AVDO}_{2}$ entre $1,3-3,0 \mu \mathrm{moL} / \mathrm{mL}$; 4) Hiperemia: LOI normal y $\mathrm{AVDO}_{2}$ reducidas $(<1,3$ $\mu \mathrm{moL} / \mathrm{mL})^{111}$. Hasta hace poco tiempo esta clasificación y el cálculo de las AVDL han sido utilizados por múltiples autores $^{3,26,70,104,105,107,110,111}$, incluido nuestro grupo ${ }^{101,124,126,128}$. 
No obstante, dadas las particularidades del transporte del lactato desde el parénquima encefálico hasta la sangre yugular, en el momento actual el valor de esta clasificación de la hemodinámica cerebral y el valor de las AVDL y del LOI como variables individuales deben cuestionarse.

\section{¿Son válidas las AVDL y el LOI en la monitorización del metabolismo cerebral?}

El cálculo de las AVDL y del LOI surgió como otra potencial aplicación del principio de Fick al encéfalo y en los últimos años estos parámetros han sido utilizados en la clínica por múltiples autores como un sistema de monitorización de la isquemia cerebral ${ }^{3,26,70,101,104,105,107,110,111,124,126,128}$. Sin embargo, para que estas cuantificaciones fueran válidas debería cumplirse la premisa que exige el principio de Fick: el elemento a cuantificar debe acceder al compartimento hemático por difusión simple. En el encéfalo, la BHE impide el intercambio libre de iones y moléculas orgánicas entre el plasma sanguíneo y el tejido nervioso, lo que asegura un medio extracelular de composición química estable y una protección frente a cambios bruscos en la concentración de solutos plasmáticos. A excepción del $\mathrm{O}_{2} \mathrm{y}$ del $\mathrm{CO}_{2}$, cualquier molécula o ión plasmático atraviesa las células endoteliales de la BHE por medio de transportadores específicos, lo que permite un control muy preciso y finamente regulado de dicho transporte. Incluso el agua no atraviesa la BHE por difusión simple, sino que utiliza una serie de canales específicos (aguaporinas) 4,77,138,141,142. Por tanto, los niveles de lactato que se cuantifican a nivel del bulbo de la yugular dependen de la acción de sistemas de transporte específicos $^{32,33,53,103}$, potencialmente inhibibles ${ }^{33,103}$, por lo que pueden no reflejar la situación real del metabolismo cerebral. Esto explica las discrepancias que se observan al determinar de forma simultánea la concentración extracelular de lactato cerebral (técnicas de microdiálisis) y las AVDL ${ }^{102,144}$.

Por otra parte, no todo el lactato producido en situación de isquemia cerebral deriva al compartimento vascular como elemento residual, sino que parte se consume por las propias células productoras y parte se transfiere a células adyacentes para ser utilizado como substrato energético ${ }^{76}$. Además, la simple medición del lactato puede confundir situaciones metabólicamente antagónicas como son un incremento del metabolismo tisular con la isquemia cerebral, ya que en ambos casos existe una elevación en la producción de lactato cerebral. El diagnóstico diferencial entre ambos procesos exige la medición simultánea de piruvato y el cálculo del índice lactato/piruvato ${ }^{55}$. De acuerdo con estos principios podemos afirmar que las AVDL y el LOI no deberían utilizarse como sistemas de monitorización del metabolismo anaerobio cerebral.

\section{ASPECTOS PRÁCTICOS A CONSIDERAR EN EL USO CLÍ- NICO DE LAS TÉCNICAS DE OXIMETRÍA YUGULAR}

\section{¿Ofrecen estas técnicas una información relevante para el manejo clínico de los pacientes neurocríticos?}

Un aspecto importante a considerar es si las técnicas de oximetría yugular aportan algún beneficio adicional en el diagnóstico y tratamiento de los pacientes neurocríticos. Debemos recordar que incluso en la versión más reciente de las guías de práctica clínica de la BTF el único parámetro que se recomienda monitorizar de forma obligada en los pacientes con un TCE grave y TC cerebral patológica es la PIC ${ }^{139}$. Este hecho, junto con las críticas de algunos autores $^{72}$, nos conducen a reflexionar sobre este punto. Los elementos clave sobre los que se sustentan algunas críticas respecto a la utilización de las técnicas de oximetría yugular son que su uso no mejora el pronóstico del paciente y que en ocasiones la información que nos ofrecen es ambigua o discordante con la información que cabría esperar por las características de determinados pacientes o de determinadas lesiones.

A pesar de que recientemente J. Cruz ${ }^{19}$ ha afirmado que la monitorización del $\mathrm{CEO}_{2}$ y de la $\mathrm{SjO}_{2}$ mejoran el pronóstico de los pacientes, la repercusión pronóstica del uso de estas técnicas de monitorización sólo podría determinarse de forma inequívoca a partir de la práctica de un estudio controlado con distribución aleatoria de los pacientes, consiguiendo que el resto de variables (tipo de lesión, edad y fenómenos intercurrentes) fueran homogéneas. Sin embargo, este estudio ideal no es factible en la práctica clínica diaria. Posiblemente, el error más común al valorar la repercusión pronóstica del uso de las técnicas de oximetría yugular ha sido no aceptar que, por si misma, su aplicación no va a mejorar el pronóstico, al igual que ocurre con cualquier otro sistema de monitorización, sino que va a ofrecer una información adicional que puede conducirnos a cambiar actitudes o modificar las medidas terapéuticas, las cuales sí pueden contribuir a cambiar el resultado final del paciente. Algunos autores enfatizan que los valores de la $\mathrm{SjO}_{2}$ en los pacientes con un TCE no deben ser un objetivo terapéutico, sino que este parámetro debe actuar como una guía terapéutica ${ }^{137}$. Por otra parte, el hecho de que en ocasiones no comprendamos plenamente la información que nos ofrecen estas variables no indica que se trate de una información errónea, sino que posiblemente no la interpretamos de forma adecuada.

En una publicación reciente referida al uso de diversas variables o técnicas de monitorización en los pacientes neurotraumáticos, Stocchetti ${ }^{135}$, apoyándose en parámetros de medicina basada en la evidencia, responde afirmativamente a la cuestión "Should I monitor jugular venous oxygen saturation?". Las conclusiones de este autor enfa- 
tizan la importancia de monitorizar el aporte de oxígeno al cerebro en los pacientes neurotraumáticos y el papel relevante que para ello tiene en el momento actual la $\mathrm{SjO}_{2}$. Reconoce también que la utilización clínica de esta variable en ocasiones no es simple, aunque su uso puede aportar información importante que puede influir de forma clara en el manejo de los pacientes ${ }^{135}$.

La utilización clínica correcta de las técnicas de oximetría yugular exige que la información que nos ofrezcan sea fiable, por lo que es fundamental que se tengan en cuenta una serie de aspectos metodológicos básicos. Además, deben conocerse las indicaciones, limitaciones y potenciales complicaciones asociadas a estas técnicas de monitorización. Su uso clínico debe dirigirse fundamentalmente a intentar mantener valores de $\mathrm{SjO}_{2}$ por encima de un determinado umbral. Sin embargo, frente a valores de $\mathrm{SjO}_{2}$ "supranormales" debería realizarse un diagnóstico diferencial de las diferentes causas etiológicas. Por otra parte, la existencia de valores de $\mathrm{SjO}_{2}$ elevados podría permitir, en algunos casos, un uso más intenso de ciertas medidas terapéuticas potencialmente lesivas como la hiperventilación. Por último, el cálculo de las $\mathrm{AVDO}_{2}$ puede ofrecernos una información adicional sobre el estado de los mecanismos de control del FSC.

\section{Consideraciones metodológicas al utilizar técnicas de oximetría yugular para estimar el valor del FSC}

Además de conocer el origen y significado de estos parámetros básicos, para utilizar las $\mathrm{AVDO}_{2}$, el $\mathrm{CEO}_{2}$ o la $\mathrm{SjO}_{2}$ como sistemas de estimación del FSC deben cumplirse unas condiciones indispensables: 1) que el consumo metabólico cerebral de oxígeno sea constante en el momento de las determinaciones y 2) que no existan problemas metodológicos. Esto nos obliga a realizar una serie de consideraciones metodológicas que deberán ser tenidas en cuenta al utilizar estas variables en el contexto de cualquier tipo de paciente neurocrítico:

\section{1) Elección de la vena yugular a cateterizar}

El drenaje de las venas yugular interna derecha e izquierda puede diferir de manera significativa, por lo que el primer aspecto a considerar es en qué vena yugular se debe insertar el catéter vascular. En general suele existir un mayor drenaje venoso cerebral a través de la vena yugular interna del lado derecho, puesto que recibe sangre predominantemente a partir del seno longitudinal superior $\mathrm{y}$, por tanto, del córtex cerebral ${ }^{44}$. De acuerdo con estos conceptos clásicos el catéter debería insertarse siempre en la vena yugular derecha. Sin embargo, Gibbs y col. ${ }^{45}$ objetivaron que en individuos normales la concentración de oxígeno y glucosa era prácticamente la misma cuando se recogían muestras simultáneas de ambas yugulares, mientras que las diferencias se acentuaban en gran medida si existían lesiones unilaterales. Atendiendo a estos resultados, en un periodo inicial se recomendaba que en los pacientes con una lesión encefálica difusa el catéter yugular debía colocarse en el lado derecho, mientras que en presencia de lesiones focales considerables el catéter debía colocarse en el lado del hemisferio más afectado ${ }^{110}$. No obstante, estudios posteriores determinaron que el predominio en el drenaje venoso cerebral podía ser muy variable ${ }^{5}$ y que muestras hemáticas obtenidas en una u otra vena yugular podían ofrecer valores distintos con diferencias clínicamente relevantes en el manejo de los pacientes neurocríticos ${ }^{72,84,85,136}$.

Diversos autores han analizado los valores de la $\mathrm{SjO}_{2}$ obtenidos de forma simultánea en pacientes a los que se les ha implantado un catéter retrógrado en ambas venas yugulares $9,72,84,85,89,136$. Mientras que algunos autores ${ }^{9}$ han confirmado los hallazgos del estudio clásico de Gibbs y col. ${ }^{45}$, otros han demostrado que existían diferencias estadísticamente significativas y clínicamente relevantes $(>10 \%)$ entre los valores obtenidos en ambos bulbos yugulares $^{72,84,85,136}$. Los resultados de estos estudios indican que la elección de la vena yugular a cateterizar debía individualizarse en cada caso.

En el momento actual se practican diversas maniobras para determinar el predominio venoso de cada paciente. La maniobra más común es valorar la repercusión de la compresión manual de cada yugular sobre la $\mathrm{PIC}^{30}$. El mayor aumento de PIC indica que a través de aquella vena yugular tiene lugar de forma preferente el drenaje venoso cerebral. Otro método que también se utiliza para seleccionar la yugular "dominante" es el análisis de la morfología del foramen yugular en la TC cerebral $^{85,136}$. En este caso debe seleccionarse la yugular que corresponda al foramen yugular de mayores dimensiones. La vena yugular dominante también puede localizarse por técnicas ultrasónicas, con una buena correlación con los métodos anteriores sin la necesidad de practicar una TC cerebral o provocar aumentos de la $\mathrm{PIC}^{13,135}$. Sin embargo, un aspecto a tener en cuenta es que incluso la cateterización de la yugular predominante no asegura que no puedan pasar desapercibidos episodios de desaturación detectables en la yugular contralateral $^{13,72,85}$.

\section{2) Método de colocación del catéter yugular}

Para la cateterización percutánea de la vena yugular, en nuestro centro utilizamos la técnica descrita por Goetting ${ }^{49}$, que constituye una modificación del método de Mostert ${ }^{86}$, según la cual el paciente debe permanecer en decúbito supino y con la cabeza algo rotada hacia el lado opuesto al punto de punción ${ }^{101}$. Este tipo de colocación facilita el proceso, puesto que provoca cierta distensión venosa. El punto 
de introducción de la aguja se localiza en el ángulo superior del triángulo de Sédillot, formado por el borde posterior del fascículo esterno-mastoideo, el borde anterior del fascículo cleido-occipital y el borde superior de la clavícula. La localización de la vena yugular se realiza con la aguja del catéter a colocar, acoplada a una jeringa de 10 cc de capacidad; el grado de inclinación de la aguja debe ser de aproximadamente $45^{\circ}$. La dirección adecuada, para la cateterización ascendente, es hacia el pabellón auricular ipsilateral ${ }^{101}$. Esta técnica resulta igualmente útil para la colocación de un catéter simple, a través del cual se obtendrán muestras hemáticas puntuales, como para la colocación de un catéter de fibra óptica que permite lecturas continuas de la $\mathrm{SjO}_{2}$.

\section{3) Correcta situación del extremo proximal del catéter yugular}

Otra de las condiciones básicas que requieren las técnicas de oximetría yugular es que las muestras de sangre venosa tengan un origen exclusivamente cerebral, por lo que el catéter debe introducirse hasta alcanzar el bulbo de la yugular. A pesar de que en los trabajos pioneros en la introducción de estas técnicas se consideraba que una muestra de sangre yugular, obtenida de forma percutánea por debajo de la apófisis mastoides, era representativa de sangre exclusivamente cerebral, Goetting ${ }^{49}$ señaló que la colocación de un catéter retrógrado hasta el bulbo de la yugular reducía al mínimo la contaminación extracerebral. Los estudios efectuados a este respecto determinaron que a nivel del bulbo de la yugular sólo un 2-3\% (7\% como máximo) de la sangre tenía un origen extracerebral, procediendo de las meninges y de la órbita ${ }^{71,134}$. Por debajo de esta situación el porcentaje de sangre extracerebral se incrementa de manera significativa ${ }^{135}$.

Robertson y col. ${ }^{111}$ objetivaron que cuando la punta del catéter se situaba $3 \mathrm{~cm}$ por debajo del bulbo de la yugular, el porcentaje de sangre extracerebral ascendía hasta el $17 \%$. Chieregato y col. ${ }^{9}$ también observaron que el valor de la $\mathrm{SjO}_{2}$ era significativamente más elevado cuando al paciente se le extraían muestras hemáticas a nivel yugular medio (quinta vértebra cervical) que cuando el extremo distal del catéter se encontraba a nivel del seno petroso inferior. Además, ambos valores también fueron significativamente mayores que los que estos autores obtenían en el bulbo de la yugular'. El aspecto más interesante de este estudio es que los valores de normalidad asumidos a partir de los estudios de Gibbs y Datsur ${ }^{27,46}$ coinciden con los valores medios obtenidos por Chieregato y col. en las tres posiciones yugulares ${ }^{9}$. Esto sugiere que posiblemente en los estudios clásicos las muestras se habían extraído de un punto situado algo por debajo del bulbo de la yugular.

\section{4) ¿Catéteres simples o sensores de fibra óptica?}

La colocación de un catéter yugular simple, sin la incorporación de sistemas de fibra óptica, constituye una posibilidad económica y al alcance de cualquier UCI. El principal inconveniente de este método es que sólo permite mediciones periódicas, por lo que pueden pasar desapercibidos episodios puntuales de caída de la $\mathrm{SjO}_{2}$. Valadka y col. ${ }^{140}$ han objetivado que muchos episodios de desaturación tienen una duración menor a una hora, pudiendo ser difíciles de detectar si sólo disponemos de una lectura de la $\mathrm{SjO}_{2}$ cada 8 o 6 horas. La utilización de catéteres de fibra óptica permite obtener lecturas continuas de la $\mathrm{SjO}_{2}$. Sin embargo, el principal inconveniente de estos sistemas es su fiabilidad, por lo que su uso obliga a considerar múltiples aspectos técnicos (posición de la punta del catéter, señal de calidad de la lectura...) y a validar muchos de los resultados obtenidos mediante extracciones manuales adicionales. A pesar de que existen autores que afirman que estos sistemas proporcionan información válida en un porcentaje bajo del tiempo de monitorización ${ }^{67}$, otros describen resultados más favorables ${ }^{74}$.

\section{5) Controles radiológicos}

La localización tan precisa de la punta del catéter obliga a realizar, en todos los casos, un estudio radiológico de control antes de proceder a la obtención de las muestras ${ }^{135}$. En una Rx cervical lateral, la punta del catéter debe localizarse aproximadamente al mismo nivel y por delante de la apófisis mastoides ${ }^{49,58}$. Otro elemento de referencia que con frecuencia se utiliza en la literatura es el disco vertebral situado entre $\mathrm{C} 1$ y $\mathrm{C} 2$. En este caso el extremo distal del catéter yugular debería encontrarse por encima de este nivel. La adquisición de una dilatada experiencia en la colocación de catéteres yugulares retrógrados no justifica la omisión de esta prueba ya que, como hemos comprobado en un número no despreciable de casos, una introducción aparentemente suficiente no siempre se corresponde con una localización correcta de la punta del catéter. En la serie de Gemma y col. ${ }^{43}$, un estudio radiológico efectuado inmediatamente después de la implantación del catéter yugular objetivó que en 39 de 172 casos el extremo distal del catéter no estaba correctamente situado en el bulbo de la yugular. En 11 ocasiones el catéter presentaba un bucle en el interior de la vena yugular; en cuatro, el catéter se había desviado hacia otro vaso venoso cervical y en un paciente se había canulado el plexo vertebral; en 10 ocasiones el catéter no había alcanzado el bulbo yugular y en 13 casos lo había sobrepasado ${ }^{43}$.

Por otra parte, también hemos observado que las movilizaciones del paciente pueden modificar la situación de la punta del catéter, especialmente si la monitorización se prolonga durante muchos días y si se utilizan sensores de fibra óptica. Por este motivo es recomendable que se reali- 
cen controles radiológicos de forma periódica. Una maniobra utilizada por algunos autores es "marcar" el punto de entrada del catéter, lo que constituye una referencia de seguridad adicional.

\section{6) Mantenimiento del catéter yugular}

Una vez colocado, el catéter yugular requiere una perfusión continua de suero fisiológico heparinizado, infundido a una velocidad suficiente para mantener la permeabilidad de la vía. También puede utilizarse una válvula antireflujo y realizar lavados periódicos, sin perfusión continua, con una solución heparinizada. Esta vía debe reservarse de forma exclusiva para la extracción de muestras hemáticas. El riesgo de trombosis se reduce al no administrar fármacos ni suplementos de potasio a través de la vía. En los casos en que se requiera una monitorización prolongada, y para reducir el riesgo de infecciones, el catéter debería sustituirse cada 5 - 7 días ${ }^{101}$.

\section{Indicaciones y contraindicaciones de la medición de las $\mathrm{AVDO}_{2}, \mathrm{CEO}_{2} \mathrm{y} \mathrm{SjO}_{2}$}

En el ámbito clínico, a pesar de que las técnicas de oximetría yugular se han utilizado fundamentalmente en el manejo de pacientes con un TCEG, la posibilidad de estudio de los fenómenos patogénicos y de la eficacia de las medidas terapéuticas hacen que también puedan aplicarse a otras patologías neurológicas agudas, especialmente en las que predominen las lesiones difusas. Por ello, la monitorización de estos parámetros puede ser muy útil en pacientes en coma por una hemorragia subaracnoidea (HSA) no traumática $\mathrm{y}$, en general, será de utilidad en todos aquellos casos en que sea necesaria la determinación del acoplamiento entre el flujo sanguíneo y el consumo metabólico cerebral de oxígeno.

La monitorización de la $\mathrm{SjO}_{2}$ se utiliza en un gran número de pacientes sometidos a intervenciones cardiovasculares de gran envergadura en las que se aplica hipotermia. La medición continua de este parámetro se ha mostrado especialmente útil durante las fases de recalentamiento del paciente, periodo en el que suelen detectarse el mayor número de desaturaciones ${ }^{11,15,60,129}$. Una rápida detección y corrección de las caídas de la $\mathrm{SjO}_{2}$ puede ayudar a prevenir las secuelas neuropsicológicas que con frecuencia presentan este tipo de pacientes ${ }^{16,59}$. Además de la cirugía cardiovascular, cada vez es más frecuente la monitorización de la $\mathrm{SjO}_{2}$ en ciertas intervenciones neuroquirúrgicas. En este contexto, la monitorización continua de la $\mathrm{SjO}_{2}$ resulta especialmente útil en aquellas intervenciones en las que el paciente requiera ser sometido a una hiperventilación prolongada o a períodos de hipotensión arterial controlada. Tales maniobras pueden reducir de forma significativa el FSC, condicionando una hipoxia cerebral oliguémica. El principal inconveniente que existe durante la monitorización neuroquirúrgica es que la movilización de la cabeza del paciente debe realizarse con precaución para no mover el extremo proximal del catéter yugular.

La monitorización de la $\mathrm{SjO}_{2}$ también resulta de gran utilidad en pacientes neurocríticos con hipertensión intracraneal refractaria a los que se aplica hipotermia como medida terapéutica de segundo nivel, especialmente durante la fase de recalentamiento. La práctica de tests de autorregulación y vasoreactividad cerebral constituye otra indicación adicional, ya que estos tests requieren la utilización de un método de estimación del FSC.

La colocación de catéteres yugulares estaría contraindicada en los pacientes con fracturas cervicales, por la imposibilidad de rotar con seguridad el cráneo, o en los casos de coagulopatía. Esta segunda contraindicación es común a cualquier sistema de monitorización invasiva que podamos aplicar a los pacientes críticos. La traqueotomía sería una contraindicación relativa, ya que incrementa el riesgo de infección del catéter yugular.

\section{Limitaciones de las $\mathrm{AVDO}_{2}, \mathrm{CEO}_{2} \mathrm{y} \mathrm{SjO}_{2}$}

Respecto a las limitaciones de estos parámetros como métodos de estimación del FSC deben mencionarse una serie de factores que pueden influir en su interpretación. En primer lugar, es conocido que en situación de alcalosis respiratoria la saturación de $\mathrm{O}_{2}$ yugular puede hallarse falsamente elevada, ya que la curva de disociación de la oxihemoglobina se desplaza hacia la izquierda (efecto Bohr). En estas condiciones tanto las $\mathrm{AVDO}_{2}$ como el $\mathrm{CEO}_{2}$ y la $\mathrm{SjO}_{2}$ podrían indicar un estado de hiperemia, cuando en realidad existe un compromiso en la liberación de $\mathrm{O}_{2}{ }_{2}^{23}$. Esta misma situación podría ocurrir en casos de hipotermia importante o de otras situaciones que incrementen la afinidad entre la hemoglobina y el oxígeno.

Las $\mathrm{AVDO}_{2}, \mathrm{CEO}_{2}$ y la $\mathrm{SjO}_{2}$ reflejan la extracción de oxígeno global del cerebro, por lo que pueden pasar desapercibidos acontecimientos metabólicos regionales ${ }^{140}$ (Fig. 4). Sin embargo, se ha demostrado que en las lesiones encefálicas difusas los cambios de flujo globales son más importantes que los cambios regionales, especialmente si el paciente está hiperventilado ${ }^{95}$. También debemos señalar, que el flujo sanguíneo de la fosa posterior contribuye de forma insignificante en la sangre venosa que drena a través del bulbo de la yugular. Por ello, en las mediciones de la extracción global de oxígeno no se recibe una información adecuada sobre el flujo sanguíneo del cerebelo ni del tronco del encéfalo ${ }^{23}$.

En los pacientes politraumatizados, en los que puede existir una anemia aguda, ya hemos comentado que la 


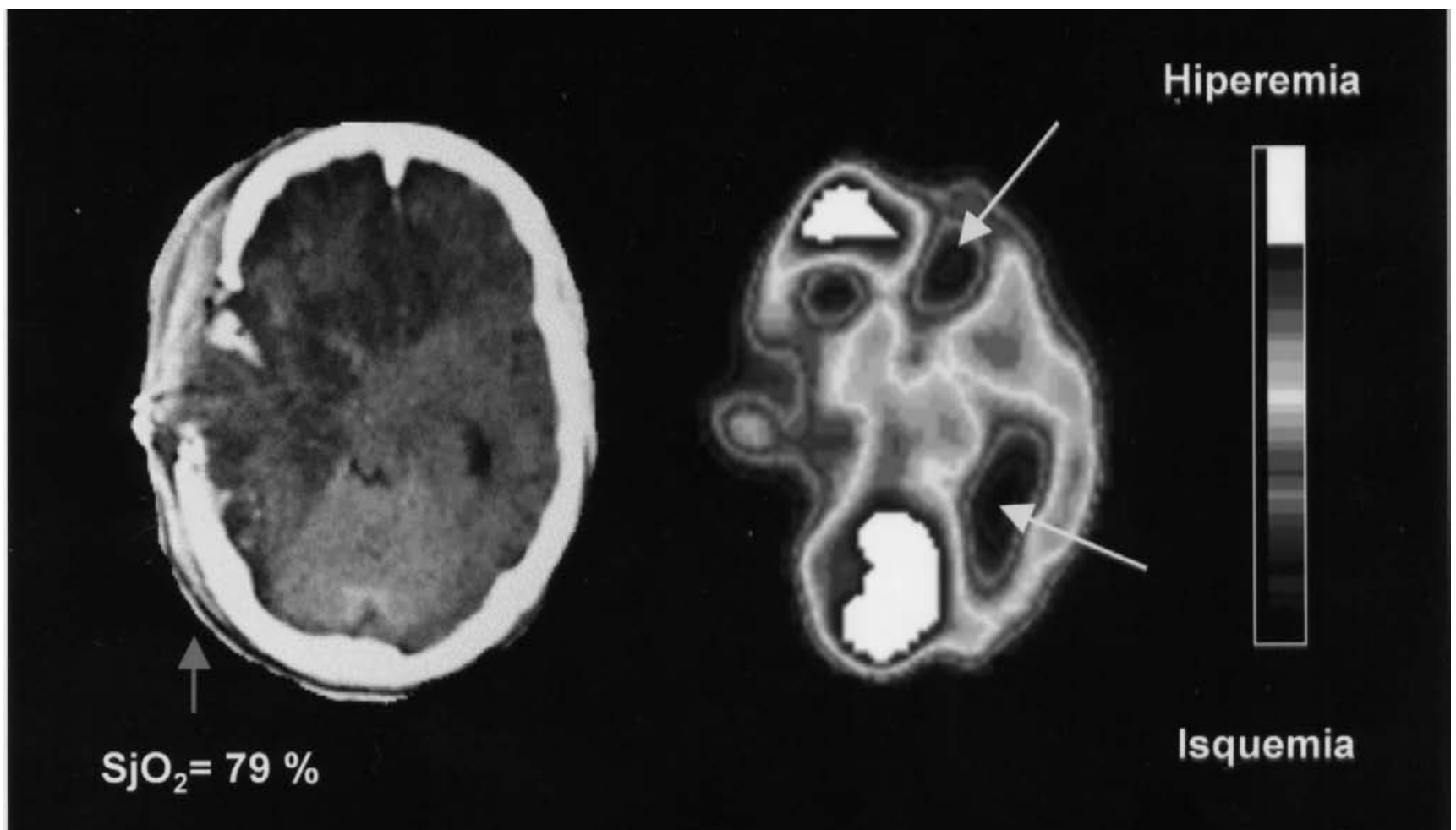

Figura 4. Paciente con valores de SjO2 elevados en el contexto de una lesión cerebral postraumática en la que existe una importante heterogeneidad en el flujo sanguineo de las diferentes regiones cerebrales. Los valores de las regiones hiperémicas pueden ponderarse con los de las regiones isquémicas, por lo que la SjO2 puede ofrecer valores normales o elevados. Las flechas blancas señalan áreas de importante reducción del FSC.

disminución de la hemoglobina conlleva un decremento en el contenido de oxígeno arterial, mientras que las $\mathrm{AVDO}_{2}$ pueden ser falsamente normales. Para evitar este artefacto, en caso de anemia importante deberían calcularse el $\mathrm{CEO}_{2}$ y la $\mathrm{SjO}_{2}$ en lugar de las $\mathrm{AVDO}_{2}$. Además, es un hecho bien conocido que un paciente puede presentar alteraciones de la oxigenación cerebral, a pesar de tener una PPC normal ${ }^{115,116}$. Tales acontecimientos pueden ocurrir en cualquier momento y los cálculos puntuales de las AVDO, pueden no detectarlos. La monitorización continua de la $\mathrm{SjO}_{2}$ a través de sensores de fibra óptica podría ayudar a establecer un diagnóstico rápido de estos fenómenos y permitir la aplicación de maniobras terapéuticas que los revirtieran.

Por último, debe tenerse en cuenta que un incremento en la $\mathrm{SjO}_{2}$ por encima del $75 \%$ puede reflejar un aumento en el FSC, pero también puede ser el resultado de una disminución no acoplada del $\mathrm{CMRO}_{2}$. Por este motivo, el valor aislado de la $\mathrm{SjO}_{2}$ no permite diferenciar entre situaciones opuestas como son la hiperemia o el infarto tisular extenso. El diagnóstico diferencial entre ambos fenómenos exige el cálculo simultáneo de la saturación de la oxihemoglobina arterial y de otros parámetros que ofrezcan información sobre el metabolismo cerebral.

\section{Complicaciones asociadas a las técnicas de oximetría yugular}

Las complicaciones asociadas a la utilización de las técnicas de oximetría yugular son extremadamente bajas ${ }^{135}$. Entre ellas se han descrito punciones accidentales de vasos venosos, arteriales o linfáticos cervicales, infecciones y trombosis de la vena yugular. Además, la colocación del catéter yugular requiere una cierta rotación cefálica, por lo que en pacientes con hipertensión intracraneal esta rotación puede obstruir temporalmente el drenaje venoso cerebral, provocando un incremento adicional de la PIC. También se ha planteado la posibilidad de que la introducción de un catéter en un vaso relativamente pequeño pueda comprometer su luz y, por tanto, aumentar la resistencia al flujo, especialmente en pacientes de corta edad ${ }^{42,49}$. No obstante, al igual que otros autores ${ }^{48}$, hemos observado que la colocación de la vía no condiciona modificaciones significativas de la tensión arterial o de la PIC si se tienen en cuenta una serie de precauciones: paciente correctamente sedado y analgesiado, cama algo incorporada y mínima rotación cefálica $^{101}$

El tiempo medio de permanencia del catéter yugular en nuestros pacientes suele ser de unos 5 días. Una vez reti- 
rado se realiza de forma rutinaria un cultivo de la punta del catéter. Hasta el momento actual, la obtención de cultivos positivos ha sido excepcional ${ }^{101}$. Creemos que esto se debe al corto período de tiempo de utilización de la vía y al hecho de que ésta se reserva exclusivamente para la extracción de muestras. Sin embargo, otros autores presentan índices de colonización/infección más elevados $(15 \%)^{72}$, aunque estos hallazgos han sido motivo de debate en la literatura ${ }^{137}$. La crítica fundamental que han recibido estos autores es que colonización no implica infección (hemocultivos negativos) y en los dos casos en los que el paciente presentó una bacteriemia los microorganismos crecieron en más de un catéter, por lo que no podía establecerse cual de ellos había sido el responsable de la infección ${ }^{137}$.

La incidencia de punciones accidentales de la arteria carótida oscila en la literatura entre un $1-4,5 \% \%^{12,74}$. En nuestro centro, en algún caso se ha puncionado la arteria carótida durante la colocación de la vía, sin otra repercusión que la aparición de un hematoma a nivel local. Después de varios años de monitorización rutinaria en los pacientes con un TCE grave o moderado, y a pesar de que en la literatura aparecen casos aislados de trombosis yugular, en nuestro centro no hemos observado otras complicaciones. La benignidad de esta técnica ha sido confirmada también por otros autores $\mathrm{s}^{43,135}$.

En un estudio realizado específicamente a determinar la incidencia de complicaciones relacionadas con la colocación y manipulación de los catéteres yugulares retrógrados, Gemma y col. ${ }^{43}$ implantaron un total de 172 catéteres en 126 pacientes y observaron que en 20 casos (12\%) se produjo una punción en la arteria carótida durante la implantación del catéter, aunque en ninguno de los pacientes presentó repercusiones; durante la implantación de otro catéter se puncionó un vaso linfático. Los autores de este estudio practicaron controles radiológicos inmediatos en todos los casos, objetivando que en el $23 \%$ de los catéteres su extremo distal no se encontraba correctamente emplazado en el bulbo de la yugular ${ }^{43}$. También practicaron estudios ultrasónicos precoces y al cabo de una semana, encontraron que en tres casos existían signos de trombosis venosa ${ }^{43}$. En dos de estos tres casos los signos de trombosis habían desaparecido al cabo de una semana. Los autores concluyen que la colocación de un catéter yugular retrógrado, cuyo extremo distal alcance el bulbo de la yugular, es un procedimiento simple y seguro, asociado a una incidencia muy reducida de complicaciones relevantes ${ }^{43}$.

\section{Incidencia de episodios de isquemia e "hiperemia" en los pacientes con un TCE. Valor pronóstico de ${\mathrm{la} \mathrm{SjO}_{2}}_{2}$}

En los TCE, la incidencia de episodios de caída de la $\mathrm{SjO}_{2}$ por debajo del umbral de isquemia puede ser muy variable, en función del punto de corte seleccionado (50\% versus
55\%), del sistema de monitorización utilizado (continuo versus discontinuo) y de los criterios diagnósticos exigidos por los diferentes autores. En algunos casos se registra la simple caída de la $\mathrm{SjO}_{2}$, independientemente de la duración del episodio ${ }^{72}$. En ocasiones se exige que la duración del episodio sea mayor de 10 minutos $^{51,106}$, mientras que otros autores cuantifican el porcentaje de tiempo de permanencia de la $\mathrm{SjO}_{2}$ por debajo del umbral isquémico, en relación con el tiempo total de registro válido de esta variable ${ }^{74}$. Por último, algunos autores cuantifican el número o porcentaje de pacientes que han presentado descensos de la $\mathrm{SjO}_{2}$ en algún momento de su curso evolutivo ${ }^{1}$. Con estas connotaciones, la incidencia de episodios isquémicos registrada en la literatura en los pacientes neurotraumáticos oscila entre un $3,4 \%$ y un $50 \% 0^{1,14,72,74}$.

En el estudio de Cormio y col. ${ }^{14}$, en el que se incluyeron 450 pacientes con un TCEG, se constató que el 4,9\% de los pacientes presentaron caídas de la $\mathrm{SjO}_{2}$ por debajo del $55 \%$. Estos episodios ocurrieron fundamentalmente durante el primer día de ingreso del paciente. En cambio, el 19,1\% de los pacientes presentaron valores de $\mathrm{la}_{\mathrm{SjO}}>75 \%{ }^{14}$. Los valores elevados de la $\mathrm{SjO}_{2}$ se encontraron de forma excepcional durante el primer día después del traumatismo, aunque la incidencia aumentó de forma progresiva durante los 4 días posteriores ${ }^{14}$. En general, la mayor parte de pacientes con un TCE presentan valores de $\mathrm{SjO}_{2}$ comprendidos dentro del rango de normalidad aceptado para este tipo de pacientes. Les siguen en frecuencia, aunque con incidencias variables, las situaciones en las que se detectan valores de $\mathrm{SjO}_{2}>75 \%$ y en menos del $10 \%$ de las ocasiones se suelen detectar valores de $\mathrm{SjO}_{2}<50-55 \%$. Cuando estos últimos ocurren suelen ser más frecuentes durante las fases precoces del traumatismo ${ }^{14,28,72,145}$.

Diversos estudios han objetivado que la presencia de episodios de desaturación de la $\mathrm{SjO}_{2}$ por debajo del umbral isquémico se asocia a un peor pronóstico ${ }^{14,51,75,106,130}$. Sin embargo, esta asociación resulta muy evidente cuando el paciente presenta caídas repetidas de $1 \mathrm{a} \mathrm{SjO}_{2}$ por debajo del $50 \%$ durante periodos de más de 10 minutos $^{51}$. Valores de $\mathrm{SjO}_{2}$ por encima del $75 \%$ también se han asociado a una mayor mortalidad y peor resultado funcional ${ }^{14,75,130}$, por lo que algunos autores sugieren que en los pacientes con un TCE deberían utilizarse algoritmos de actuación frente a descensos o aumentos anómalos de esta variable ${ }^{75}$.

\section{Algoritmo de actuación frente a caídas de la $\mathrm{SjO}_{2}$}

En el curso evolutivo de los pacientes neurocríticos existen múltiples situaciones en las que pueden producirse descensos bruscos de la $\mathrm{SjO}_{2}$, sugerentes de isquemia cerebral. Con el objetivo de identificar la etiología de estos descensos, en la literatura se han propuesto una serie de protocolos de actuación general. Se trata de protocolos 
escalonados en los que se van descartando problemas de forma secuencial. A continuación proponemos un algoritmo de actuación frente a caídas en los valores de la $\mathrm{SjO}_{2}$ por debajo del umbral de isquemia:

- En primer lugar deben descartarse problemas técnicos: si el paciente es portador de un catéter de fibra óptica debe confirmarse la validez de la lectura, analizando la señal de calidad de lectura en el monitor, o recalibrando el sistema. $\mathrm{Si}$ es necesario deben comprobarse los valores con una extracción yugular manual. Si los valores persisten bajos:

- Deben analizarse los parámetros relacionados con el aporte de oxígeno al cerebro: concentración de hemoglobina y $\mathrm{PaO}_{2}$, para descartar una anemia o una hipoxemia. El diagnóstico de estas situaciones obligaría a transfundir al paciente o a incrementar la $\mathrm{FiO}_{2}$ respectivamente. Sin embargo, si estos parámetros son normales, debe pasarse al siguiente estadio diagnóstico:

- En este punto deben valorarse aquellos factores que influyen en el FSC: PPC, $\mathrm{PaCO}_{2}$ y PIC. Si la PPC está por debajo de $60 \mathrm{mmHg}$, deberemos aumentar la PAM; si el paciente está hiperventilado en exceso, deberá incrementarse la $\mathrm{PaCO}_{2}$; si existe una hipertensión intracraneal, intensificaremos las maniobras terapéuticas.

Cuando todos los aspectos anteriores han sido valorados y los valores de $\mathrm{SjO}_{2}$ siguen bajos, deberíamos disminuir las demandas metabólicas del encéfalo, tratando la hipertermia o las posibles crisis comiciales, administrando hipnóticos y/o reduciendo la temperatura del enfermo. La figura 5 resume este algoritmo de actuación.

¿Cómo actuar cuando un paciente presenta valores de $\mathrm{SjO}_{2}>75 \%$ ?

A pesar de que hemos comentado que los valores de $\mathrm{SjO}_{2}>75 \%$ son patológicos, pueden deberse a procesos fisiopatológicos distintos y se asocian a un peor pronóstico, no existen protocolos de actuación bien definidos que puedan utilizarse cuando nos enfrentamos a estos valores. La mayor parte de los autores no indican cómo actúan frente a lecturas de la $\mathrm{SjO}^{2}>75 \%$ o indican de forma explícita que no aplican maniobras terapéuticas específicas frente a estos valores. Sin embargo, si la información conjunta que nos ofrecen diferentes variables sugiere o indica que los valores elevados de la $\mathrm{SjO}_{2}$ corresponden a una situación de hiperemia, y el paciente presenta una hipertensión intracraneal, podríamos incrementar la hiperventilación o utilizar con mayor seguridad otras maniobras terapéuticas que pueden inducir una vasoconstricción cerebral.
2005; 16: 301-322

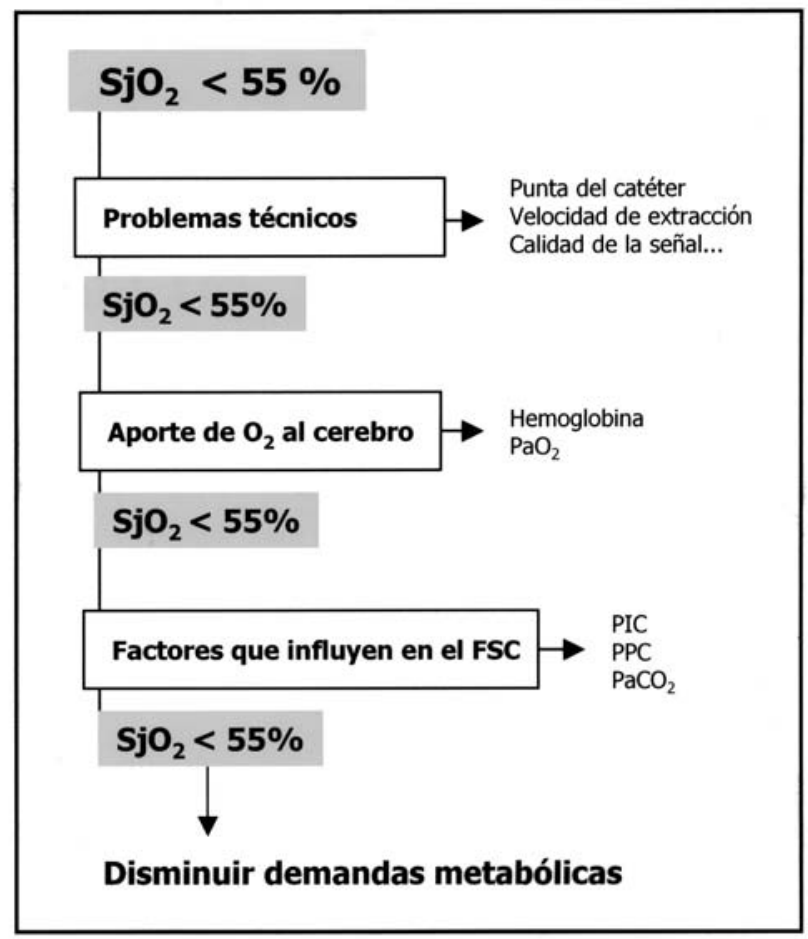

Figura 5. Algoritmo de actuación frente a la detección de valores de la $\mathrm{SjO}_{2}$ por debajo del umbral de isquemia.

\section{LAS TÉCNICAS DE OXIMETRÍA YUGULAR COMO INSTRU- MENTOS DE ESTUDIO DE LOS MECANISMOS DE CON- TROL DEL FSC}

La alteración de los mecanismos reguladores del FSC en la fase aguda de los TCE es un hecho bien conocido, aunque siguen existiendo controversias respecto a su incidencia real y significado pronóstico ${ }^{78,88,91,95,99,120,125}$. La disfunción de los mecanismos fisiológicos de autorregulación y los trastornos de la reactividad arteriolar a los cambios de presión arterial de $\mathrm{CO}_{2}\left(\mathrm{PaCO}_{2}\right)$ juegan un importante papel en estas alteraciones. La autorregulación y la reactividad vascular al $\mathrm{CO}_{2}$ constituyen mecanismos de control independientes, que pueden alterarse de forma aislada. Estudiar el estado de ambos mecanismos en determinados pacientes neurocríticos permite conocer mejor la fisiopatología de cada caso y seleccionar o descartar determinadas medidas terapéuticas. El cálculo de las $\mathrm{AVDO}_{2}$ puede utilizarse para definir el grado de preservación o alteración de los mecanismos de autorregulación y reactividad de los vasos cerebrales al $\mathrm{CO}_{2}$.

Estudio de las alteraciones de la reactividad vascular cerebral al $\mathrm{CO}_{2}$ a partir de las diferencias arterioyugulares de oxígeno

Hasta hace pocos años la hiperventilación constituía 
una de las maniobras iniciales en el tratamiento de la hipertensión intracraneal. No obstante, diversos estudios han demostrado que su uso agresivo e indiscriminado puede conducir a la isquemia cerebral ${ }^{23,38,98}$. La hiperventilación puede reducir el flujo sanguíneo hasta el límite de la isquemia en áreas en las que la reactividad al $\mathrm{CO}_{2}$ se halla preservada $\mathrm{y}$, simultáneamente, puede incrementar el flujo sanguíneo en aquellas otras regiones en las que existe una alteración o una abolición de la reactividad al $\mathrm{CO}_{2}$ (fenómeno del robo inverso) ${ }^{38}$. Además, algunos autores han sugerido que en los TCEG existe una alteración más o menos marcada de la capacidad de las arteriolas cerebrales para responder a los cambios en la $\mathrm{PaCO}^{278,83,90,96}$. El conocimiento del grado de reactividad cerebrovascular al $\mathrm{CO}_{2}$ en el contexto de estos pacientes, puede ser de suma importancia para evaluar la potencial eficacia de medidas terapéuticas como la propia hiperventilación, los barbitúricos, el propofol u otros fármacos ${ }^{83,87,94}$.

Valorar el grado de alteración o preservación de los vasos cerebrales al $\mathrm{CO}_{2}$ implica calcular los cambios que se producen en el FSC a partir de las modificaciones en la $\mathrm{PaCO}_{2}$. El cálculo de las $\mathrm{AVDO}_{2}$ permite evaluar la reactividad cerebral al $\mathrm{CO}_{2}$, al modificar de forma controlada y transitoria los parámetros ventilatorios del paciente, partiendo de la premisa que el $\mathrm{CMRO}_{2}$ y la hemoglobina van a permanecer constantes durante la práctica del test. En esta situación, el FSC va a ser directamente proporcional al valor inverso de las $\mathrm{AVDO}_{2}\left(1 / \mathrm{AVDO}_{2}\right)^{131,132}$. Los cambios en el FSC producidos a partir de variaciones en la $\mathrm{PaCO}_{2}$, van a traducirse en cambios paralelos en las $1 / \mathrm{AVDO}_{2}, \mathrm{y}$ los valores obtenidos van a ser fiables siempre y cuando no se produzcan cambios simultáneos $>10 \%$ en la TAM del paciente ${ }^{100,121}$. A partir de estos parámetros puede calcularse la reactividad absoluta o especifica al $\mathrm{CO}_{2}$ (cambio absoluto de las $\mathrm{AVDO}_{2}$ por cada $\mathrm{mmHg}$ de cambio en la $\left.\mathrm{PaCO}_{2}\right)^{123}$ y el porcentaje de reactividad $\left(\mathrm{CO}_{2} \mathrm{R} \%\right.$ : porcentaje de incremento o decremento del FSC estimado [1/ $\mathrm{AVDO}_{2}$ ] por cada $\mathrm{mmHg}$ de cambio en la $\left.\mathrm{PaCO}_{2}\right)^{123}$. Los valores de referencia se han obtenido a partir de los resultados de diversos estudios en los que se ha calculado el porcentaje de cambio del FSC por cada $\mathrm{mmHg}$ de cambio de $\mathrm{PaCO}_{2}$ en voluntarios sanos, valores que oscilan entre 1.8 y $2.3^{65,66,80,81}$. El $\mathrm{CO}_{2} \mathrm{R} \%$ nos permite diferenciar entre una reactividad cerebrovascular al $\mathrm{CO}_{2}$ preservada $\left(\mathrm{CO}_{2} \mathrm{R} \%>\right.$ $1 \%$ ) o alterada/abolida $\left(\mathrm{CO}_{2} \mathrm{R} \% \leq 1 \%\right)^{123}$.

Estudio de las alteraciones de la autorregulación cerebral a partir de las diferencias arterio-yugulares de oxígeno

La alteración de la autorregulación en la fase aguda de los TCE es un hecho todavía más frecuente que las alteraciones de la reactividad cerebrovascular al $\mathrm{CO}_{2}{ }^{100,118,121,123} \mathrm{y}$ juega un importante papel en la fisiopatología del aumento del volumen sanguíneo cerebral y de la hipertensión intracraneal ${ }^{57,95}$. En los últimos años algunos autores han afirmado que en la fase aguda de los TCE el objetivo primordial debe ser el mantenimiento de cifras elevadas de $\mathrm{PPC}^{112-114}$. No obstante, en los pacientes con una alteración y/o abolición de la autorregulación, esta maniobra puede ser conflictiva. El aumento de la PPC en este subgrupo de pacientes puede incrementar el volumen sanguíneo cerebral y sobrecargar la presión capilar, facilitando fenómenos de edema cerebral por disrupción de la barrera hematoencefálica. Por otra parte, cuando los mecanismos de autorregulación cerebral se hallan alterados o abolidos, el encéfalo se hace altamente sensible a los episodios de hipotensión arterial. En esta situación, la hipotensión puede precipitar una situación isquémica, dado que el FSC se reduce paralelamente al descenso de las cifras de la tensión arterial.

La autorregulación también es un elemento mediador importante en los mecanismos de acción de ciertas terapias que se utilizan en el control de la PIC. Algunos autores han demostrado que el manitol es mucho más efectivo en pacientes con una autorregulación intacta ${ }^{87}$. Después de la administración de manitol se produce un aumento del FSC, que parece ser mucho más significativo en aquellos pacientes con una autorregulación cerebral alterada o abolida ${ }^{87}$, lo cual podría explicar el "efecto rebote" sobre la PIC que la administración de un bolus de manitol provoca en algunos pacientes. El conocimiento del estado de la autorregulación en la fase aguda de los TCE, puede ayudar a establecer protocolos de tratamiento individualizados y evitar maniobras terapéuticas inadecuadas en este heterogéneo grupo de pacientes.

Las $\mathrm{AVDO}_{2}$ también permiten estudiar el estado de la autorregulación cerebral, al estimar cambios en el FSC inducidos a partir de cambios en la TAM del paciente. Después de haber obtenido unas $\mathrm{AVDO}_{2}$ basales $\left(\mathrm{AVDO}_{2 \mathrm{~B}}\right)$, se administra fenilefrina y se incrementa gradualmente la TAM del paciente en un $25 \%$ aproximadamente ${ }^{123}$. Después de conseguir una TAM estable (al cabo de 10-20 min), se practica una segunda extracción arterial y yugular para el cálculo de las $\mathrm{AVDO}_{2}\left(\mathrm{AVDO}_{2 \mathrm{~F}}\right)$. A partir de estos datos, se calcula el cambio porcentual de FSC respecto a las condiciones basales ${ }^{123}$. Si los mecanismos de autorregulación están preservados, los cambios en el FSC deben ser mínimos. Aunque no existen unos límites de "normalidad" claramente establecidos, de acuerdo con los criterios de Enevoldsen y Jensen ${ }^{38}$ cambios en el FSC estimado de \pm un $20 \%$ indican que la autorregulación se encuentra preservada, mientras que los valores superiores al $20 \%$ son indicativos de una autorregulación alterada o abolida.

La figura 6 resume los cálculos a realizar para valorar el estado de los mecanismos de autorregulación y reactividad 


\section{A - Reactividad vascular cerebral al $\mathrm{CO}_{2}$}

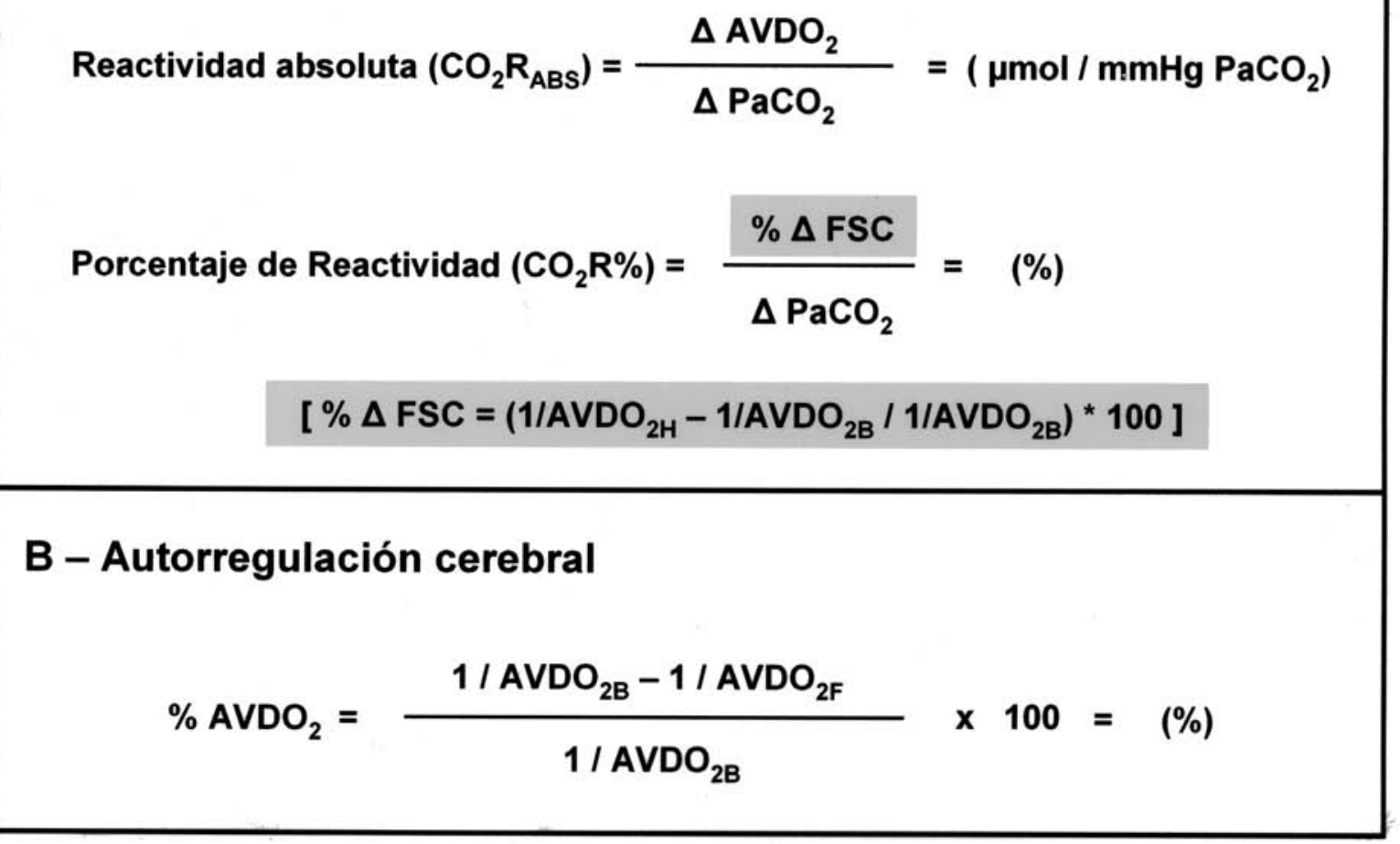

Figura 6. Parámetros y fórmulas a utilizar para determinar el estado de la reactividad de los vasos cerebrales al CO ${ }_{2}$ (parte superior) y de la autorregulación (parte inferior) en los pacientes neurocríticos a partir de las AVDO como método de estimación del FSC. (B) Valores basales; (H) Valores después de la hiperventilación; (F) Valores finales.

de los vasos cerebrales al $\mathrm{CO}_{2}$ a partir de las técnicas de oximetría yugular como método de estimación del FSC.

\section{Conclusiones}

Los pacientes neurocríticos, y en especial los que han presentado un TCE, son susceptibles de presentar un gran número de lesiones secundarias que pueden empeorar su pronóstico. El elemento común de todas ellas suele ser el desarrollo o la potenciación de la isquemia cerebral, lo que justifica la necesidad de una monitorización multimodal en este tipo de pacientes. Las técnicas de oximetría yugular se han utilizado durante años con este objetivo. $\mathrm{La} \mathrm{SjO}_{2}$ constituye el parámetro más simple y el más utilizado en la práctica clínica para estimar el FSC y valorar la idoneidad entre el aporte y el consumo cerebral de oxígeno. En el contexto de los TCE, el objetivo fundamental de la monitorización de la $\mathrm{SjO}_{2}$ es mantener cifras $>55 \%$. Esto implica modificar, cuando sea oportuno, la aplicación de ciertas medidas terapéuticas. No obstante, lecturas de la $\mathrm{SjO}_{2}>75 \%$ no indican necesariamente una situación de seguridad para el paciente, ya que estos valores pueden ser compatibles con situaciones fisiopatológicas muy distintas que requieran maniobras terapéuticas inversas. Para esclarecer el significado de estas lecturas elevadas de la $\mathrm{SjO}_{2}$ se requiere el uso simultáneo de otros métodos de monitorización como el Doppler transcraneal o la determinación de variables regionales o locales como la presión tisular de oxígeno o los parámetros metabólicos que ofrecen las técnicas de microdiálisis cerebral. Por último, debemos considerar que la monitorización de la $\mathrm{SjO}_{2}$ por si misma no mejora el pronóstico de los pacientes, al igual que ocurre con la monitorización de cualquier otra variable (incluida la PIC). Sin embargo, la medición de la $\mathrm{SjO}_{2}$ o de otras variables obtenidas a partir de las técnicas de oximetría yugular (a excepción de las AVDL y del LOI) pueden ayudarnos a optimizar y racionalizar las medidas terapéuticas que aplicamos y de esta forma influir en el resultado final de nuestros pacientes.

\section{Agradecimientos}

Agradecemos la eficaz colaboración del personal de enfermería de la UCI de Traumatología en el registro de 
las numerosas variables que derivan de la monitorización multimodal aplicada a los pacientes neurotraumáticos.

Este trabajo ha sido financiado en parte por la beca FIS 03/0153 del Fondo de Investigación Sanitaria y por la Red Temática de Investigación Cooperativa de Enfermedades Neurológicas (Red CIEN, C3/06, Nodo 11, Hospital Universitario Vall d'Hebron).

\section{Bibliografía}

1. Abdo, C.A., Figueredo, M.J., Castellanos, G.R., et al.: Monitorización de la saturación yugular de oxígeno y la isquemia cerebral en el paciente neurocrítico. Rev Neurol 2001; 33: 511-513.

2. Alberico, A.M., Ward, J.D., Choi, S.C., et al.: Outcome after severe head injury. Relationship to mass lesions, diffuse injury, and ICP course in pediatric and adult patients. J Neurosurg 1987; 67: 648-656.

3. Artru, F., Dailler, F., Burel, E., et al.: Assessment of jugular blood oxygen and lactate indices for detection of cerebral ischemia and prognosis. J Neurosurg Anesthesiol 2004; 16: 226-231.

4. Badaut, J., Lasbennes, F., Magistretti, P.J., et al.: Aquaporins in brain: distribution, physiology, and pathophysiology. J Cereb Blood Flow Metab 2002; 22: 367-378.

5. Beards, S.C., Yule, S., Kassner, A., et al.: Anatomical variation of cerebral venous drainage: the theoretical effect on jugular bulb blood samples. Anaesthesia 1998; 53: 627-633.

6. Bulger, E.M., Nathens, A.B., Rivara, F.P., et al.: Management of severe head injury: institutional variations in care and effect on outcome. Crit Care Med 2002; 30: 1870-1876.

7. Chesnut, R.M.: Secondary brain insults after head injury: clinical perspectives. New Horiz 1995; 3: 366-375.

8. Chesnut, R.M., Marshall, L.F., Klauber, M.R., et al.: The role of secondary brain injury in determining outcome from severe head injury. J Trauma 1993; 34: 216-222.

9. Chieregato, A., Calzolari, F., Trasforini, G., et al.: Normal jugular bulb oxygen saturation. J Neurol Neurosurg Psychiatry 2003; 74: 784-786.

10. Cold, G.E.: Cerebral blood flow in acute head injury. The regulation of cerebral blood flow and metabolism during the acute phase of head injury, and its significance for therapy. Acta Neurochir Suppl (Wien) 1990; 49: 1-64.

11. Cook, D.J., Oliver, W.C., Jr., Orszulak, T.A., et al.: A prospective, randomized comparison of cerebral venous oxygen saturation during normothermic and hypothermic cardiopulmonary bypass. J Thorac Cardiovasc Surg 1994; 107 : 1020-1028.

12. Coplin, W.M., OKeefe, G.E., Grady, M.S., et al.: Thrombotic, infectious, and procedural complications of the jugular bulb catheter in the intensive care unit. Neurosurgery 1997; 41: 101-107.

13. Cormio, M., Robertson, C.S.: Ultrasound is a reliable method for determining jugular bulb dominance. J Neurosurg Anesthesiol 2001; 13: 250-254.

14. Cormio, M., Valadka, A.B., Robertson, C.S.: Elevated jugular venous oxygen saturation after severe head injury. $\mathrm{J}$ Neurosurg 1999; 90: 9-15.

15. Croughwell, N.D., Frasco, P., Blumenthal, J.A., et al.: Warming during cardiopulmonary bypass is associated with jugular bulb desaturation. Ann Thorac Surg 1992; 53: 827832.

16. Croughwell, N.D., Newman, M.F., Blumenthal, J.A., et al.: Jugular bulb saturation and cognitive dysfunction after cardiopulmonary bypass. Ann Thorac Surg 1994; 58: 17021708.

17. Cruz, J.: Continuous versus serial global cerebral hemometabolic monitoring: Applications in acute brain trauma. Acta Neurochir Suppl (Wien) 1988; 42: 35-39.

18. Cruz, J.: Cerebral oxygenation. Monitoring and management. Acta Neurochir Suppl (Wien) 1993; 59: 86-90.

19. Cruz, J.: The first decade of continuous monitoring of jugular bulb oxyhemoglobin saturation: management strategies and clinical outcome. Crit Care Med 1998; 26: 344-351.

20. Cruz, J., Allen, S.J., Miner, M.E.: Hypoxic insults in acute brain injury. Crit Care Med 1985; 13: 284.

21. Cruz, J., Hoffstad, O.J., Jaggi, J.L.: Cerebral lactateoxygen index in acute brain injury with acute anemia: Assessment of false versus true ischemia. Crit Care Med 1994; 22: 1465-1470.

22. Cruz, J., Jaggi, J.L., Hoffstad, O.J.: Cerebral blood flow and oxygen consumption in acute brain injury with acute anemia: An alternative for the cerebral metabolic rate of oxygen consumption. Crit Care Med 1993; 21: 1218-1224.

23. Cruz, J., Miner, M.E.: Modulating cerebral oxygen delivery and extraction in acute traumatic coma. En Miner ME, Wagner KA (eds). Neurotrauma. Treatment, rehabilitation, and related issues. Boston; Butterworths, 1986; pp. 5572.

24. Cruz, J., Miner, M.E., Allen, S.J., et al.: Continuous monitoring of cerebral oxygenation in acute brain injury: Injection of mannitol during hyperventilation. J Neurosurg 1990; 73: 725-730.

25. Cruz, J., Raps, E.C., Hoffstad, O.J., et al.: Cerebral oxygenation monitoring. Crit Care Med 1993; 21: 12421246.

26. Cruz, J., Zager, E.L., Schnee, C.L., et al.: Failure of jugular lactate determinations to disclose cerebral ischemia in posttraumatic cerebral infarction: case report. J Trauma 1993; 35: 805-807.

27. Datsur, D.K., Lane, M.H., Hansen, D.B., et al.: Effects of aging on cerebral circulation and metabolism in man. En Birren JE, Butler RN, Greenhouse SW, et al. (eds). Human aging. A biological and behavioral study. Washington; US Government Printing Office, 1963; pp. 59-76.

28. De Deyne, C., Decruyenaere, J., Calle, P., et al.: Analy- 
sis of very early jugular bulb oximetry data after severe head injury: implications for the emergency management? Eur J Emerg Med 1996; 3: 69-72.

29. De Deyne, C., Van Aken, J., Decruyenaere, J., et al.: Jugular bulb oximetry: review on a cerebral monitoring technique. Acta Anaesthesiol Belg 1998; 49: 21-31.

30. Dearden, N.M.: Benefits and pitfalls of jugular bulb venous oxygen saturation monitoring. En Tsubokawa T, Marmarou A, Robertson C, et al. (eds). Neurochemical monitoring in the intensive care unit. Tokyo; Springer-Verlag, 1995; pp. 87-97.

31. Dearden, N.M., Midgley, S.: Technical considerations in continuous jugular venous oxygen saturation measurement. Acta Neurochir Suppl (Wien) 1993; 59: 91-97.

32. Deuticke, B.: Monocarboxylate transport in erythrocytes. J Membr Biol 1982; 70: 89-103.

33. Deuticke, B., Beyer, E., Forst, B.: Discrimination of three parallel pathways of lactate transport in the human erythrocyte membrane by inhibitors and kinetic properties. Biochim Biophys Acta 1982; 684: 96-110.

34. DeWitt, D.S., Jenkins, L.W., Prough, D.S.: Enhanced vulnerability to secondary ischemic insults after experimental traumatic brain injury. New Horiz 1995; 3: 376-383.

35. Díaz-Reganon, G., Minambres, E., Holanda, M., et al.: Usefulness of venous oxygen saturation in the jugular bulb for the diagnosis of brain death: report of 118 patients. Intensive Care Med 2002; 28: 1724-1728.

36. Edvinsson, L., Mackenzie, E.T., McCulloch, J.: Disturbed cerebral autoregulation. En Edvinsson L (ed). Cerebral blood flow and metabolism. New York; Raven Press, 1993; pp. 599-609.

37. Eisenberg, H.M., Frankowski, R.F., Contant, C.F., et al.: High-dose barbiturate control of elevated intracranial pressure in patients with severe head injury. J Neurosurg 1988; 69: 15-23.

38. Enevoldsen, E.M., Jensen, F.T.: Autoregulation and $\mathrm{CO}_{2}$ responses of cerebral blood flow in patients with acute severe head injury. J Neurosurg 1978; 48: 698-703.

39. Fakhry, S.M., Trask, A.L., Waller, M.A., et al.: Management of brain-injured patients by an evidence-based medicine protocol improves outcomes and decreases hospital charges. J Trauma 2004; 56: 492-499.

40. Fick, A.: Ueber die messung des blutquantums in den herzventrikeln. Stiz ber Physik-Med Ges Wúrzburg. Sitz ber Physik-Med Ges Wúrzburg 1870; 2: 16-28.

41. Garlick, R., Bihari, D.: The use of intermittent and continuous recordings of jugular venous blood oxygen saturation in the unconscious patient. Scand J Clin Lab Invest 1987; 47: 47-52.

42. Gayle, M.O., Frewen, T.C., Armstrong, R.F., et al.: Jugular venous bulb catheterization in infants and children. Crit Care Med 1989; 17: 385-388.

43. Gemma, M., Beretta, L., De Vitis, A., et al.: Complica- tions of internal jugular vein retrograde catheterization. Acta Neurochir Suppl (Wien) 1998; 71: 320-323.

44. Gibbs, E.L., Gibbs, F.A.: The cross section areas of the vessels that from the torcular and the manner is which flow is distributed to the right and to the left lateral sinus. Anac Rec 1934; 59: 419-426.

45. Gibbs, E.L., Lennox, W.G., Gibbs, F.A.: Bilateral internal jugular blood. Comparaison of A-V differences, oxygendextrose ratios and respiratory quotients. Am J Psychiat 1945; 102: 184-190.

46. Gibbs, E.L., Lennox, W.G., Nims, L.F., et al.: Arterial and cerebral venous blood arterial-venous differences in man. J Biol Chem 1942; 144: 325-332.

47. Goeting, M.G.: Validation of the jugular bulb catheter for cerebral venous monitoring. Neurology 1990; 40: 254.

48. Goetting, M.G., Preston, G.: Effect of jugular bulb catheterization on intracranial pressure. En Hoff JT, Betz AL (eds). Intracranial pressure VII. Berlin; Springer-Verlag, 1989; pp. 116-118.

49. Goetting, M.G., Preston, G.: Jugular bulb catheterization: Experience wth 123 patients. Crit Care Med 1999; 18: 1220-1223.

50. Golding, E.M., Robertson, C.S., Bryan, R.M., Jr.: The consequences of traumatic brain injury on cerebral blood flow and autoregulation: a review. Clin Exp Hypertens 1999; 21: 299-332.

51. Gopinath, S.P., Robertson, C.S., Contant, C.F., et al.: Jugular venous desaturation and outcome after head injury. J Neurol Neurosurg Psychiatry 1994; 57: 717-723.

52. Gupta, A.K.: Monitoring the injured brain in the intensive care unit. J Postgrad Med 2002; 48: 218-225.

53. Halestrap, A.P.: Transport of pyruvate and lactate into human erythrocytes. Evidence for the involvement of the chloride carrier and a chloride-independent carrier. Biochem J 1976; 156: 193-207.

54. Hans, P.: Venous oxygen saturation in the jugular bulb. En Vincent JL (ed). Update in intensive care and emergency medicine 14. Berlin; Sringer-Verlag, 1991; pp. 516-521.

55. Hillered, L., Persson, L.: Microdialysis for neurochemical monitoring in human brain injuries. En Tsubokawa T, Marmarou A, Robertson C, et al. (eds). Neurochemical monitoring in the intensive care unit. Tokyo; Springer-Verlag, 1995; pp. 59-63.

56. Hirayama, T., Katayama, Y., Tsubokawa, T.: Jugular bulb oximetry in patients with cerebral arteriovenous malformation. En Tsubokawa T, Marmarou A, Robertson C, et al. (eds). Neurochemical monitoring in the intensive care unit. Microdialysis, jugular venous oximetry, and near-infrared spectroscopy. Tokyo; Springer-Verlag, 1995; pp. 146-150.

57. Jaggi, J.L., Obrist, W.D., Gennarelli, T.A., et al.: Relationship of early cerebral blood flow and metabolism to outcome in acute head injury. J Neurosurg 1990; 72: 176-182.

58. Jakobsen, M., Enevoldsen, E.: Retrograde catheteriza- 
tion of the right internal jugular vein for serial measurements of cerebral venous oxygen content. Technical note. J Cereb Blood Flow Metab 1989; 9: 717-720.

59. Kadoi, Y., Saito, S., Goto, F., et al.: Decrease in jugular venous oxygen saturation during normothermic cardiopulmonary bypass predicts short-term postoperative neurologic dysfunction in elderly patients. J Am Coll Cardiol 2001; 38: 1450-1455.

60. Kawahara, F., Kadoi, Y., Saito, S., et al.: Slow rewarming improves jugular venous oxygen saturation during rewarming. Acta Anaesthesiol Scand 2003; 47: 419-424

61. Keller, E., Steiner, T., Fandino, J., et al.: Jugular venous oxygen saturation thresholds in trauma patients may not extrapolate to ischemic stroke patients: lessons from a preliminary study. J Neurosurg Anesthesiol 2002; 14: 130-136.

62. Kett-White, R., Hutchinson, P.J., Czosnyka, M., et al.: Multi-modal monitoring of acute brain injury. Adv Tech Stand Neurosurg 2002; 27: 87-134.

63. Kety, S.S., Schimdt, C.F.: The determination of cerebral blood flow in man by the use of nitrous oxide in low concentrations. Am J Physiol 1945; 143: 53-66.

64. Kety, S.S., Schimdt, C.F.: The nitrous oxide method for the quantitative-determination of cerebral blood flow in man: theory, procedure and normal values. J Clin Invest 1948; 27: 476-483.

65. Kety, S.S., Schmidt, C.F.: Effects of altered arterial tensions of carbon dioxide and oxygen on cerebral blood flow and cerebral oxygen consumption of normal young men. J Clin Invest 1948; 27: 484-492.

66. Kety, S.S., Schmidt, C.F.: The effects of active and passive hyperventilation on cerebral blood flow, cerebral oxygen consumption, cardiac output, and blood pressure of normal young men. J Clin Invest 1948; 25: 107-119.

67. Kiening, K.L., Unterberg, A.W., Bardt, T.F., et al.: Monitoring of cerebral oxygenation in patients with severe head injuries: brain tissue $\mathrm{PO}_{2}$ versus jugular vein oxygen saturation. J Neurosurg 1996; 85: 751-757.

68. Krogh, A., Lindhard, J.: Measurements of the blood flow through the lungs of man. Scand Arch Physiol 1912; 27: $100-125$

69. Langfitt, T.W., Obrist, W.D.: Occlusive cerebrovascular disease. En Wilkins RH, Rengachary SS (eds). Neurosurgery. New York; McGraw-Hill Book Company, 1985; pp. 11671173.

70. Larson, C.P., Jr., Ehrenfeld, W.K., Wade, J.G., et al.: Jugular venous oxygen saturation as an index of adequacy of cerebral oxygenation. Surgery 1979; 62: 31-39.

71. Lassen, N.A., Lane, M.H.: Validity of internal jugular blood for study of cerebral blood flow and metabolism. J Appl Physiol 1961; 16: 313-320.

72. Latronico, N., Beindorf, A.E., Rasulo, F.A., et al.: Limits of intermittent jugular bulb oxygen saturation monitoring in the management of severe head trauma patients.
Neurosurgery 2000; 46: 1131-1138.

73. Le Roux, P.D., Newell, D.W., Lam, A.M., et al.: Cerebral arteriovenous oxygen difference: a predictor of cerebral infarction and outcome in patients with severe head injury. J Neurosurg 1997; 87: 1-8.

74. MacMillan, C.S., Andrews, P.J.: Cerebrovenous oxygen saturation monitoring: practical considerations and clinical relevance. Intensive Care Med 2000; 26: 1028-1036.

75. MacMillan, C.S., Andrews, P.J., Easton, V.J.: Increased jugular bulb saturation is associated with poor outcome in traumatic brain injury. J Neurol Neurosurg Psychiatry 2001; 70: 101-104.

76. Magistretti, P.J., Pellerin, L.: Astrocytes Couple Synaptic Activity to Glucose Utilization in the Brain. News Physiol Sci 1999; 14: 177-182.

77. Marmarou, A.: Pathophysiology of traumatic brain edema: current concepts. Acta Neurochir Suppl (Wien) 2003; 86: 7-10.

78. Marmarou, A., Wachi, A.: Blood volume responsivity to ICP change in head injuried patients. En Hoff JT, Betz AL (eds). Intracranial pressure VII. Berlin; Springer-Verlag, 1989; pp. 688-690.

79. Marshall, L.F., Bowers, S.H.A.: Medical management of intracranial pressure. En Cooper PR (ed): Head injury. Baltimore; Williams \& Wilkins, 1987; pp. 177-196.

80. McHenry, L.C.J., Slocum, H.C., Bivens, H.E., et al.: Hyperventilation in awake and anesthetized man. Effects on cerebral blood flow and cerebral metabolism. Arch Neurol 1965; 12: 270-277.

81. McHenry, L.C.J., Slocum, H.C., Hayes, G.J.: The effects of hyperventilation on the cerebral circulation and metabolism. Trans Ann Neurol Asso 1964; 89: 223-225.

82. McKinley, B.A., Parmley, C.L., Tonneson, A.S.: Standardized management of intracranial pressure: a preliminary clinical trial. J Trauma 1999; 46: 271-279.

83. Messeter, K., Nordström, C.H., Sundbärg, G., et al.: Cerebral hemodynamics in patients with acute severe head trauma. J Neurosurg 1986; 64: 231-237.

84. Metz, C., Holzschuh, M., Bein, T., et al.: Jugular bulb monitoring of cerebral oxygen metabolism in severe head injury: accuracy of unilateral measurements. Acta Neurochir Suppl (Wien) 1998; 71: 324-327.

85. Metz, C., Holzschuh, M., Bein, T., et al.: Monitoring of cerebral oxygen metabolism in the jugular bulb: reliability of unilateral measurements in severe head injury. J Cereb Blood Flow Metab 1998; 18: 332-343.

86. Mostert, J.W., Kenny, G.M., Murphy, G.P.: Safe placement of central venous catheter into internal jugular vein. Arch Surg 1970; 101: 431-432.

87. Muizelaar, J.P., Lutz, H.A., Becker, D.P.: Effect of manitol on ICP and CBF and correlation with pressure autoregulation in severely head-injured patients. J Neurosurg 1984; 61: 700-706. 
88. Muizelaar, J.P., Marmarou, A., DeSalles, A.A., et al.: Cerebral blood flow and metabolism in severely head-injuried children. Part 1: Relationship with GCS score, outcome, ICP, and PVI. J Neurosurg 1989; 71: 63-71.

89. Muizelaar, J.P., Schroder, M.L.: Overview of monitoring of cerebral blood flow and metabolism after severe head injury. Can J Neurol Sci 1994; 21: S6-S11.

90. Muizelaar, J.P., Van der Poel, H.G., Kontos, H.A., et al.: Pial arteriolar vessel diameter and $\mathrm{CO}_{2}$ reactivity during prolonged hyperventilation in the rabbit. J Neurosurg 1988; 69: 923-927.

91. Muizelaar, J.P., Ward, J.D., Marmarou, A., et al.: Cerebral blood flow and metabolism in severely head-injuried children. Part 2: Autoregulation. J Neurosurg 1989; 71: 72-76.

92. Myerson, A., Halloran, R.D., Hirsch, H.L.: Technic for obtaining blood the internal jugular vein and internal carotid artery. Arch Neurol Psychiat 1927; 17: 807-808.

93. Nakajima, T., Ohsumi, H., Kuro, M.: Accuracy of continuous jugular bulb venous oximetry during cardiopulmonary bypass. Anesth Analg 1993; 77: 1111-1115.

94. Nordström, C.H., Messeter, K., Sundbärg, G., et al.: Cerebral blood flow, vasoreactivity, and oxygen consumption during barbiturate therapy in severe traumatic brain lesions. J Neurosurg 1988; 68: 424-431.

95. Obrist, W.D., Langfitt, T.W., Jaggi, J.L., et al.: Cerebral blood flow and cerebral metabolism in comatose patients with acute head injury. Relationship to intracranial hypertension. J Neurosurg 1984; 61: 241-253.

96. Overgaard, J., Tweed, W.A.: Cerebral circulation after head injury. Part 1: Cerebral blood flow after closed head injury with emphasis on clinical correlations. J Neurosurg 1974; 41: 531-541.

97. Overgaard, J., Tweed, W.A.: Cerebral circulation after head injury. Part 2: The effects of traumatic brain edema. J Neurosurg 1976; 45: 292-300.

98. Patel, P.M.: Hyperventilation as a therapeutic intervention: do the potential benefits outweigh the known risks? J Neurosurg Anesthesiol 1993; 5: 62-65.

99. Peenninger, E.G., Reith, A., Breiting, D., et al.: Early changes of intracranial pressure, perfusion pressure, and blood flow after acute head injury. Part 1: An experimental study of the underlying pathophysiology. J Neurosurg 1989; 70: 10871096.

100. Poca, M. A.: Monitorización de la hemodinámica cerebral en los traumatismos craneoencefálicos graves a partir de las diferencias arterio-yugulares de oxígeno. Universidad Autónoma de Barcelona. Barcelona. 1991. Tesis Doctoral.

101. Poca, M.A,. Sahuquillo, J., Chasampi, A., et al.: Monitorización de la hemodinámica cerebral en los traumatismos craneoencefálicos graves a partir de las diferencias arterioyugulares de oxígeno. Fundamentos, metodología e indicaciones. Neurocirugía 1993; 4: 285-298.

102. Poca, M.A., Sahuquillo, J., Mena, M.P., Vilalta, A.,
Ibañez, J., Garnacho, A.: Arterio-jugular differences of lactate (AVDL) and the lactate-oxygen index are not reliable indicators of increased brain anaerobic metabolism in traumatic brain injury. J Neurotrauma (in press).

103. Poole, R.C., Halestrap, A.P.: Transport of lactate and other monocarboxylates across mammalian plasma membranes. Am J Physiol 1993; 264: C761-C782.

104. Raichle, M.E., Posner, J.B., Plum, F.: Cerebral blood flow during and after hyperventilation. Arch Neurol 1970; 23 : 394-404.

105. Rehncrona, S., Rosén, I., Siesjö, B.K.: Brain lactic acidosis and ischemic cell damage: 1 . biochemistry and neurophysiology. J Cereb Blood Flow Metab 1981; 1: 297-311.

106. Robertson, C.: Desaturation episodes after severe head injury: influence on outcome. Acta Neurochir Suppl (Wien) 1993; 59: 98-101.

107. Robertson, C.S.: Anaerobic metabolism within the brain: its relationship to brain failure in head-injuried patients. En Bihari D, Holaday JW (eds). Update in intensive care and emergency medicine 9. Brain failure. Berlin; Springer-Verlag, 1989; pp. 85-102.

108. Robertson, C.S.: $\mathrm{SjVO}_{2}$ monitoring in head-injured patients. En Tsubokawa T, Marmarou A, Robertson C, et al. (eds). Neurochemical monitoring in the intensive care unit. Microdialysis, jugular venous oximetry, and near-infrared spectroscopy. Tokyo; Springer-Verlag, 1995; pp. 153-159.

109. Robertson, C.S., Contant, C.F., Gokaslan, Z.L., et al.: Cerebral blood flow, arteriovenous oxygen difference, and outcome in head injured patients. J Neurol Neurosurg Psychiatry 1992; 55: 594-603.

110. Robertson, C.S., Grossman, R.G., Goodman, J.C., et al.: The predictive value of cerebral anaerobic metabolism with cerebral infarction after head injury. J Neurosurg 1987; 67: 361-368

111. Robertson, C.S., Narayan, R.K., Gokaslan, Z.L., et al.: Cerebral arteriovenous oxygen difference as an estimate of cerebral blood flow in comatose patients. J Neurosurg 1989; 70: 222-230.

112. Rosner, M.J., Rosner, S.D.: Cerebral perfusion pressure management of head injury. En Avezaat CJJ, van Eijndhoven JHM, Maas AIR, et al. (eds). Intracranial pressure VIII. Berlin, Heidelberg; Springer-Verlag, 1994; pp. 540-543.

113. Rosner, M.J., Rosner, S.D.: CPP management II: optimization of CPP or vasoparalysis does not exist in the living brain. En Nagai H, Kamiya K, Ishii S (eds). Intracranial pressure IX. Tokyo; Springer-Verlag, 1994; pp. 222-224.

114. Rosner, M.J., Rosner, S.D., Johnson, A.H.: Cerebral perfusion pressure: management protocol and clinical results. J Neurosurg 1995; 83: 949-962.

115. Sahuquillo, J., Amorós, S., Poca, M.A., et al.: Coexistence of regional cerebral hypoxia with normal or hyperemic brain detected by global monitoring methods. Analysis of apparently contradictory findings based on the Siggaard- 
Andersen model of tissue hypoxia. Acta Neurochir Suppl (Wien) 2002; 81: 303-305.

116. Sahuquillo, J., Amorós, S., Santos, A., et al.: Does an increase in cerebral perfusion pressure always mean a better oxygenated brain? A study in head-injured patients. Acta Neurochir Suppl (Wien) 2000; 76: 457-462.

117. Sahuquillo, J., Amorós, S., Santos, A., et al.: False autoregulation (pseudoautoregulation) in patients with severe head injury. Its importance in CPP management. Acta Neurochir Suppl (Wien) 2000; 76: 485-490.

118. Sahuquillo, J., Baguena, M., Campos, L., et al.: $\mathrm{CO}_{2}$ reactivity and autoregulation in severe head injury: bedside assessment by relative changes in arteriojugular differences of oxygen. En Tsubokawa T, Marmarou A, Robertson C, et al. (eds). Neurochemical monitoring in the intensive care unit. Tokyo; Springer-Verlag, 1995; pp. 180-189.

119. Sahuquillo, J., Biestro, A., Mena, M.P., et al.: Medidas de primer nivel en el tratamiento de la hipertensión intracraneal en el paciente con un traumatismo craneoencefálico grave. Propuesta y justificación de un protocolo. Neurocirugía 2002; 13: 78-100.

120. Sahuquillo, J., Castaño, C., Vilalta, J., et al.: Reactividad vascular cerebral al $\mathrm{CO}_{2}$ en la fase aguda de los traumatismos craneoencefálicos severos. Estudio preliminar de 20 casos. Neurocirugía 1991; 1: 261-268.

121. Sahuquillo, J., Munar, F., Báguena, M., Poca, M.A., Pedraza, S., Rodríguez-Baeza, A.: Evaluation of cerebrovascular $\mathrm{CO}_{2}$-reactivity and autoregulation in patients with post-traumatic diffuse brain swelling (diffuse injury III). Acta Neurochir Suppl (Wien) 1998; 71: 233-236.

122. Sahuquillo, J., Poca, M.A.: Diffuse axonal injury after head trauma. A review. Adv Tech Stand Neurosurg 2002; 27 : 23-86.

123. Sahuquillo, J., Poca, M.A., Ausina, A., et al.: Arterio-jugular differences of oxygen $\left(\mathrm{AVDO}_{2}\right)$ for bedside assessment of $\mathrm{CO}_{2}$-reactivity and autoregulation in the acute phase of severe head injury. Acta Neurochir (Wien) 1996; 138: 435444.

124. Sahuquillo, J., Poca, M.A., Garnacho, A., et al.: Is there agreement between the different methods we use in monitoring brain ischemia? A comparative study using arterio-jugular differences of oxygen $\left(\mathrm{AVDO}_{2}\right)$, oxyhemoglobin saturation $\left(\mathrm{SjO}_{2}\right)$ and cerebral extraction of oxygen $\left(\mathrm{CEO}_{2}\right)$. En Nagai H, Kamiya K, Ishii S (eds). Intracranial pressure IX. Tokyo; Springer-Verlag, 1994; pp. 42-45.

125. Sahuquillo, J., Poca, M.A., Garnacho, A., et al.: $\mathrm{CO}_{2}$ reactivity, autoregulation and hemodynamic reserve in the first 24 hours after severe head injury: bedside assessment by relative changes in $\mathrm{AVDO}_{2}$ En Nagai H, Kamiya K, Ishii S (eds). Intracranial pressure IX. Tokyo; Springer-Verlag, 1994; pp. 683-685.

126. Sahuquillo, J., Poca, M.A., Garnacho, A., et al.: Early ischaemia after severe head injury. Preliminary results in patients with diffuse brain injuries. Acta Neurochir (Wien) 1993; 122: 204-214.

127. Sahuquillo, J., Poca, M.A., Munar, F., et al.: Avances en el tratamiento de los traumatismos craneoencefálicos graves. Neurocirugía 1999; 10: 185-209.

128. Sahuquillo, J., Poca, M.A., Robles, A., et al.: Lesiones isquémicas en la fase aguda de los TCE graves (Lesiones Difusas). Neurocirugía 1992; 3: 313-322.

129. Sapire, K.J., Gopinath, S.P., Farhat, G., et al.: Cerebral oxygenation during warming after cardiopulmonary bypass. Crit Care Med 1997; 25: 1655-1662.

130. Schoon, P., Benito, M.L., Orlandi, G., et al.: Incidence of intracranial hypertension related to jugular bulb oxygen saturation disturbances in severe traumatic brain injury patients. Acta Neurochir Suppl (Wien) 2002; 81: 285287.

131. Shalit, M.N., Beller, A.J., Feinsod, M., et al.: The blood flow and oxygen consumption of the dying brain. Neurology 1970; 20: 740-748.

132. Shapiro, W., Wasserman, A.J., Patterson, J.L.Jr.: Human cerebrovascular response time to elevation of arterial carbon dioxide tension. Arch Neurol 1965; 13: 130-138.

133. Sheinberg, M., Kanter, M.J., Robertson, C.S., et al.: Continuous monitoring of jugular venous oxygen saturation in head-injured patients. J Neurosurg 1992; 76: 212-217.

134. Shenkin, H.A., Harmel, M.H., Kety, S.S.: Dinamyc anatomy of the cerebral circulation. Arch Neurol 1948; 60: 240-252.

135. Stocchetti, N.: Should I monitor jugular venous oxygen saturation? En Valadka AB, Andrews BT (eds). Neurotrauma. Evidence-based answers to common questions. New York, Stuttgart; Thieme Medical Publishers, 2004; pp. 58-61.

136. Stocchetti, N., Paparella, A., Bridelli, F., et al.: Cerebral venous oxygen saturation studied with bilateral samples in the internal jugular veins. Neurosurgery 1994; 34: 38-44.

137. Stocchetti, N., Rossi, S.: Limits of intermittent jugular bulb oxygen saturation monitoring in the management of severe head trauma patients. Neurosurgery 2001; 48: 454-456 (comment).

138. Takata, K., Matsuzaki, T., Tajika, Y.: Aquaporins: water channel proteins of the cell membrane. Prog Histochem Cytochem 2004; 39: 1-83.

139. The Brain Trauma Foundation. The American Association of Neurological Surgeons. The Joint Section on Neurotrauma Critical Care. Management and prognosis of severe traumatic brain injury. Part 1: guidelines for the management of severe traumatic brain injury. J Neurotrauma 2000; 17: 449-554.

140. Valadka, A.B., Furuya, Y., Hlatky, R., et al.: Global and regional techniques for monitoring cerebral oxidative metabolism after severe traumatic brain injury. Neurosurg 
Focus 2000; 9.

141. Venero, J.L., Machado, A., Cano, J.: Importance of aquaporins in the physiopathology of brain edema. Curr Pharm Des 2004; 10: 2153-2161.

142. Venero, J.L., Vizuete, M.L., Machado, A., et al.: Aquaporins in the central nervous system. Prog Neurobiol 2001; 63: 321-336.

143. Vespa, P.M.: Should I monitor cerebral blood flow after traumatic brain injury? En Valadka AB, Andrews BT (eds). Neurotrauma. Evidence-based answers to common questions. New York, Stuttgart; Thieme Medical Publishers, 2004; pp. 68-72.

144. Vespa, P.M., Mcarthur, D., O’Phelan, K., et al.: Persistently low extracellular glucose correlates with poor outcome 6 months after human traumatic brain injury despite a lack of increased lactate: a microdialysis study. J Cereb Blood Flow Metab 2003; 23: 865-877.

145. Vigue, B., Ract, C., Benayed, M., et al.: Early $\mathrm{SjvO}_{2}$ monitoring in patients with severe brain trauma. Intensive Care Med 1999; 25: 445-451.

146. Yokota, H., Yamamoto, Y., Nakabayashi, M., et al.: Continuous monitoring of yugular venous oxygen saturation in neurosurgical intensive care units. En Tsubokawa T, Marmarou A, Robertson C, et al. (eds). Neurochemical monitoring in the intensive care unit. Tokyo; Springer-Verlag, 1995; pp. 98-104.

Poca, M.A.; Sahuquillo, J.; Monforte, R.; Vilalta, A.: Métodos globales de monitorización de la hemodinámica cerebral en el paciente neurocrítico: fundamentos, controversias y actualizaciones en las técnicas de oximetría yugular. Neurocirugía 2005; 16: 301-322.

Correspondencia postal: M.A. Poca. Servicio de Neurocirugía Hospital Universitario Vall d'Hebron. Paseo Vall d'Hebron 119129. 08035, Barcelona. 